\title{
COMPLEX ZERO DECREASING SEQUENCES
}

\author{
Thomas Craven and George Csordas
}

\begin{abstract}
The purpose of this paper is to investigate the real sequences $\gamma_{0}, \gamma_{1}$, $\gamma_{2}, \ldots$ with the property that if $p(x)=\sum_{k=0}^{n} a_{k} x^{k}$ is any real polynomial, then $\sum_{k=0}^{n} \gamma_{k} a_{k} x^{k}$ has no more nonreal zeros than $p(x)$. In particular, the authors establish a converse to a classical theorem of Laguerre.
\end{abstract}

\section{Introduction and background information}

In the theory of distribution of zeros of polynomials, the following open problem is of central interest. Let $D$ be a subset of the complex plane. Characterize the linear transformations $T$ carrying polynomials into polynomials such that if $p$ is a polynomial (either arbitrary or restricted to a certain class of polynomials), then the polynomial $T[p]$ has at least as many zeros in $D$ as $p$ has zeros in $D$. There is an analogous problem for transcendental entire functions. (For related questions and results, see, for example, [2], [9], [13, Ch. 2,4], [14], [15, Ch. 7], [18, Ch. 3-5], and [20, Ch. 1$2]$.) In the classical setting $(D=\mathbb{R})$, the problem (solved by Pólya and Schur [24]) is to characterize all real sequences $T=\left\{\gamma_{k}\right\}_{k=0}^{\infty}, \gamma_{k} \in \mathbb{R}$ such that if a polynomial $p(x)=\sum_{k=0}^{n} a_{k} x^{k}$ has only real zeros, then the polynomial

$$
T[p(x)]=T\left[\sum_{k=0}^{n} a_{k} x^{k}\right]:=\sum_{k=0}^{n} \gamma_{k} a_{k} x^{k},
$$

also has only real zeros (see (1.4) and (1.5) below). The purpose of this paper is to attack the following more general problem. Characterize all real sequences $T=$ $\left\{\gamma_{k}\right\}_{k=0}^{\infty}, \gamma_{k} \in \mathbb{R}$ such that if $p(x)$ is any real polynomial, then

$$
Z_{c}(T[p(x)]) \leq Z_{c}(p(x))
$$

where $Z_{c}(p(x))$ denotes the number of nonreal zeros of $p(x)$, counting multiplicities. In order to facilitate the description of our results, we will first recall some definitions and terminology and review some facts that will be needed in the sequel.

Definition 1.1. A real entire function $\phi(x):=\sum_{k=0}^{\infty} \gamma_{k} x^{k} / k$ ! is said to be in the Laguerre-Pólya class, $\phi(x) \in \mathcal{L}-\mathcal{P}$, if $\phi(x)$ can be expressed in the form

$$
\phi(x)=c x^{n} e^{-\alpha x^{2}+\beta x} \prod_{k=1}^{\infty}\left(1+\frac{x}{x_{k}}\right) e^{-\frac{x}{x_{k}}}
$$

where $c, \beta, x_{k} \in \mathbb{R}, c \neq 0, \alpha \geq 0, n$ is a nonnegative integer, and $\sum_{k=1}^{\infty} 1 / x_{k}^{2}<\infty$. If $-\infty \leq a<b \leq \infty$ and if $\phi(x) \in \mathcal{L}$-P has all its zeros in $(a, b)$ (or $[a, b])$, then we will use the notation $\phi \in \mathcal{L}-\mathcal{P}(a, b)$ (or $\phi \in \mathcal{L}-\mathcal{P}[a, b])$. If $\gamma_{k} \geq 0$ (or $(-1)^{k} \gamma_{k} \geq 0$ or $-\gamma_{k} \geq 0$ )

Received November 12, 1994, revised May 3, 1995.

1991 Mathematics Subject Classification. Primary 26C10, 30D15. Secondary 30D10.

Key words and phrases: Laguerre-Pólya class, multiplier sequences, distribution of zeros of entire functions, positive definite sequences. 
for all $k=0,1,2, \ldots$, then $\phi \in \mathcal{L}-\mathcal{P}$ is said to be of type I in the Laguerre-Pólya class, and we will write $\phi \in \mathcal{L}$ - $\mathcal{P}$ I.

In order to clarify the above terminology, we remark that if $\phi \in \mathcal{L}$-PI, then $\phi \in$ $\mathcal{L}-\mathcal{P}(-\infty, 0]$ or $\phi \in \mathcal{L}-\mathcal{P}[0, \infty)$, but that an entire function in $\mathcal{L}-\mathcal{P}(-\infty, 0]$ need not belong to $\mathcal{L}$-PI. (Indeed, if $\phi(x)=\frac{1}{\Gamma(x)}$ where $\Gamma(x)$ denotes the gamma function, then $\phi(x) \in \mathcal{L}$ - $\mathcal{P}(-\infty, 0]$, but $\phi(x) \notin \mathcal{L}$ - $\mathcal{P}$ I. This can be seen, for example, by looking at the Taylor coefficients of $\phi(x)=\frac{1}{\Gamma(x)}$.)

Definition 1.2. A sequence $T=\left\{\gamma_{k}\right\}_{k=0}^{\infty}$ of real numbers is called a multiplier sequence if, whenever the real polynomial $p(x)=\sum_{k=0}^{n} a_{k} x^{k}$ has only real zeros, the polynomial $T[p(x)]=\sum_{k=0}^{n} \gamma_{k} a_{k} x^{k}$ also has only real zeros.

The following are well-known characterizations of multiplier sequences (cf. [20, pp. 29-47], [23, pp. 100-124], or [24]). A sequence $T=\left\{\gamma_{k}\right\}_{k=0}^{\infty}$ is a multiplier sequence if and only if

$$
\phi(x)=T\left[e^{x}\right]:=\sum_{k=0}^{\infty} \frac{\gamma_{k}}{k !} x^{k} \in \mathcal{L}-\mathcal{P} \mathrm{I} .
$$

Moreover, the algebraic characterization of multiplier sequences asserts that a sequence $T=\left\{\gamma_{k}\right\}_{k=0}^{\infty}$ is a multiplier sequence if and only if

$$
g_{n}(x):=\sum_{j=0}^{n}\left(\begin{array}{l}
n \\
j
\end{array}\right) \gamma_{j} x^{j} \in \mathcal{L}-\mathcal{P} \mathrm{I} \text { for all } n=1,2,3, \ldots
$$

The polynomials $g_{n}(x)$ are called the Jensen polynomials associated with the entire function $\phi(x)$ defined by (1.4).

Definition 1.3. We say that a sequence $\left\{\gamma_{k}\right\}_{k=0}^{\infty}$ is a complex zero decreasing sequence (CZDS) if

$$
Z_{c}\left(\sum_{k=0}^{n} \gamma_{k} a_{k} x^{k}\right) \leq Z_{c}\left(\sum_{k=0}^{n} a_{k} x^{k}\right)
$$

for any real polynomial $\sum_{k=0}^{n} a_{k} x^{k}$. (The acronym CZDS also will be used in the plural.)

Now it follows from (1.6) that any complex zero decreasing sequence is also a multiplier sequence. If $T=\left\{\gamma_{k}\right\}_{k=0}^{\infty}$ is a sequence of nonzero real numbers, then inequality (1.6) is equivalent to the statement that for any polynomial $p(x)=\sum_{k=0}^{n} a_{k} x^{k}, T[p]$ has at least as many real zeros as $p$ has. There are, however, CZDS which have zero terms (cf. Section 3); consequently, it may happen that $\operatorname{deg} T[p]<\operatorname{deg} p$. When counting the real zeros of $p$, the number generally increases with the application of $T$ but, in fact, may decrease due to a decrease in the degree of the polynomial. For this reason, we count nonreal zeros rather than real ones. The existence of a nontrivial CZDS is a consequence of the following theorem proved by Laguerre and extended by Pólya (see Pólya [22] or [23, pp. 314-321]). We remark that part (2) follows from (1) by a limiting argument.

Theorem 1.4. (Laguerre [20, Satz 3.2]) (1) Let $f(x)=\sum_{k=0}^{n} a_{k} x^{k}$ be an arbitrary real polynomial of degree $n$ and let $h(x)$ be a polynomial with only real zeros, none of which lie in the interval $(0, n)$. Then $Z_{c}\left(\sum_{k=0}^{n} h(k) a_{k} x^{k}\right) \leq Z_{c}(f(x))$. 
(2) Let $f(x)=\sum_{k=0}^{n} a_{k} x^{k}$ be an arbitrary real polynomial of degree $n$, let $\phi \in \mathcal{L}-\mathcal{P}$, and suppose that none of the zeros of $\phi$ lie in the interval $(0, n)$. Then the inequality $Z_{c}\left(\sum_{k=0}^{n} \phi(k) a_{k} x^{k}\right) \leq Z_{c}(f(x))$ holds.

(3) Let $\phi \in \mathcal{L}-\mathcal{P}(-\infty, 0]$. Then the sequence $\{\phi(k)\}_{k=0}^{\infty}$ is a complex zero decreasing sequence.

One of the main results of this paper (see Theorem 2.13) is the converse of Theorem 1.4 in the case that $\phi$ is a polynomial. The converse fails, in general, for transcendental entire functions. Indeed, if $p(x)$ is a polynomial in $\mathcal{L}-\mathcal{P}(-\infty, 0)$, then $\frac{1}{\Gamma(-x)}+p(x)$ and $\sin (\pi x)+p(x)$ are transcendental entire functions which generate the same sequence $\{p(k)\}_{k=0}^{\infty}$, but they are not in $\mathcal{L}-\mathcal{P}$.

For several analogues and extensions of Theorem 1.4, we refer the reader to Karlin [15, pp. 379-383], Marden [18, pp. 60-74], Obreschkoff [20, pp. 6-8, 42-47], and Weisner [27]. A sequence $\left\{\gamma_{k}\right\}_{k=0}^{\infty}$ which can be interpolated by a function $\phi \in \mathcal{L}-\mathcal{P}(-\infty, 0)$, that is, $\phi(k)=\gamma_{k}$ for $k=0,1,2, \ldots$, will be called a Laguerre multiplier sequence or a Laguerre sequence. It follows from Theorem 1.4 that Laguerre sequences are multiplier sequences. The reciprocals of Laguerre sequences are examples of sequences which are known as $\lambda$-sequences in the literature (cf. Iliev [13, Ch. 4] or Kostova [16]) and are defined as follows.

Definition 1.5. A sequence of nonzero real numbers, $\Lambda=\left\{\lambda_{k}\right\}_{k=0}^{\infty}$, is called a $\lambda$ sequence if

$$
\Lambda[p(x)]=\Lambda\left[\sum_{k=0}^{n} a_{k} x^{k}\right]:=\sum_{k=0}^{n} \lambda_{k} a_{k} x^{k}>0 \text { for all } x \in \mathbb{R}
$$

whenever $p(x)=\sum_{k=0}^{n} a_{k} x^{k}>0$ for all $x \in \mathbb{R}$.

Remark 1.6. We remark that if $\Lambda$ is a sequence of nonzero real numbers, and if $\Lambda\left[e^{-x}\right]$ is an entire function, then a necessary condition for $\Lambda$ to be a $\lambda$-sequence is that $\Lambda\left[e^{-x}\right] \geq 0$ for all real $x$. (Indeed, if $\Lambda\left[e^{-x}\right]<0$ for $x=x_{0}$, then continuity considerations show that there is a positive integer $n$ such that $\Lambda\left[(1-x / 2 n)^{2 n}+1 / n\right]<$ 0 for $x=x_{0}$.)

In $[13$, Ch. 4] (see also [16]), it was pointed out by Iliev that $\lambda$-sequences are precisely the positive definite sequences (see Theorem 1.7(2) below). There are several known characterizations of positive definite sequences (see, for example, [19, Ch. 8] and [29, Ch. 3]), which we include here for the reader's convenience.

Theorem 1.7. Let $\Lambda=\left\{\lambda_{k}\right\}_{k=0}^{\infty}$ be a sequence of nonzero real numbers, then the following are equivalent.

(1) $\Lambda$ is a $\lambda$-sequence.

(2) (Positive Definite Sequences [29, p. 132]) For any polynomial $p(x)=\sum_{k=0}^{n} a_{k} x^{k}$, $p$ not identically zero, the relation $p(x) \geq 0$ for all $x \in \mathbb{R}$ implies that

$$
\Lambda[p](1)=\sum_{k=0}^{n} \lambda_{k} a_{k}>0 .
$$

(3) (Determinant Criterion [29, p. 134])

$$
\operatorname{det}\left(\lambda_{i+j}\right)=\left|\begin{array}{cccc}
\lambda_{0} & \lambda_{1} & \cdots & \lambda_{n} \\
\lambda_{1} & \lambda_{2} & \cdots & \lambda_{n+1} \\
\vdots & \vdots & & \vdots \\
\lambda_{n} & \lambda_{n+1} & \cdots & \lambda_{2 n}
\end{array}\right|>0 \quad \text { for } n=0,1,2, \ldots
$$


(4) (The Hamburger Moment Problem [29, p. 134]) There exists a nondecreasing function $\mu(t)$ with infinitely many points of increase such that

$$
\lambda_{n}=\int_{-\infty}^{\infty} t^{n} d \mu(t) \quad \text { for } n=0,1,2, \ldots
$$

The importance of $\lambda$-sequences in our investigation stems from the fact that a necessary condition for a sequence $T=\left\{\gamma_{k}\right\}_{k=0}^{\infty}, \gamma_{k}>0$, to be a CZDS is that the sequence of reciprocals $\Lambda=\left\{\frac{1}{\gamma_{k}}\right\}_{k=0}^{\infty}$ be a $\lambda$-sequence. Thus, for example, the reciprocal of a Laguerre multiplier sequence is a $\lambda$-sequence. As our next example shows, there are multiplier sequences whose reciprocals are not $\lambda$-sequences.

Example 1.8. Let $T=\left\{1+k+k^{2}\right\}_{k=0}^{\infty}$. Then by (1.4), $T$ is a multiplier sequence, since

$$
(1+x)^{2} e^{x}=\sum_{k=0}^{\infty} \frac{1+k+k^{2}}{k !} x^{k} \in \mathcal{L}-\mathcal{P} \mathrm{I} .
$$

Next, let $\Lambda=\left\{\lambda_{k}\right\}_{k=0}^{\infty}=\left\{\frac{1}{1+k+k^{2}}\right\}_{k=0}^{\infty}$. Then a calculation shows that $\operatorname{det}\left(\lambda_{i+j}\right)$, $(i, j=0, \ldots, 3)$, is

$$
\left|\begin{array}{cccc}
1 & \frac{1}{3} & \frac{1}{7} & \frac{1}{13} \\
\frac{1}{3} & \frac{1}{7} & \frac{1}{13} & \frac{1}{21} \\
\frac{1}{7} & \frac{1}{13} & \frac{1}{21} & \frac{1}{31} \\
\frac{1}{13} & \frac{1}{21} & \frac{1}{31} & \frac{1}{43}
\end{array}\right|=-\frac{55936}{2833723113403}=-1.9739 \cdots \times 10^{-8} .
$$

Therefore, by (1.8), we conclude that $\Lambda$ is not a $\lambda$-sequence, and, a fortiori, the multiplier sequence $T$ is not a CZDS. It also is instructive to exhibit a concrete example for which inequality (1.2) fails. To this end, we set $p(x):=(x+1)^{6}\left(x^{2}+\frac{1}{2} x+\frac{1}{5}\right)$. Then a calculation shows that

$$
T[p(x)]=\frac{1}{10}(x+1)^{4}\left(730 x^{4}+785 x^{3}+306 x^{2}+43 x+2\right) .
$$

It now can be verified that $Z_{c}(T[p(x)])=4 \not \leq Z_{c}(p(x))=2$, and hence it again follows that the multiplier sequence $T$ is not a CZDS.

It should be said that the present paper supersedes our papers (cf. [4]-[8]) in which we claimed that all multiplier sequences are complex zero decreasing sequences. Unfortunately, our investigations were vitiated by our oversight that some of the theorems of our previous papers are incorrect. Piecemeal correction is not the purpose of this paper, and perhaps, at this distance of time, it is hardly desirable. The recognition of this mistake, however, has led us to develop afresh the arguments from a different point of view which has enabled us, in particular, to characterize completely those CZDS which can be interpolated by polynomials (see Theorem 2.13 of Section 2). In Section 3 , we establish the existence of CZDS which have only a finite number of nonzero terms (Theorem 3.1 and Proposition 3.5), and we prove that for certain functions $\phi \in \mathcal{L}-\mathcal{P}$, but $\phi \notin \mathcal{L}$ - $\mathcal{P}$ I, the sequence $\{\phi(k)\}_{k=0}^{\infty}$ is a CZDS (Corollary 3.3). In Section 4, we establish the existence of a class of CZDS, $\left\{\gamma_{k}\right\}_{k=0}^{\infty}$, for which the sequence $\left\{g_{k}(t)_{k=0}^{\infty}\right\}$ is a CZDS for all $t \geq \gamma_{1}$, where $g_{k}(t)=\sum_{j=0}^{k}\left(\begin{array}{c}k \\ j\end{array}\right) \gamma_{j} t^{j}$ (see Corollary 4.7). To this end, we first prove a generalization of a classical theorem of Hutchinson [12] (Theorem 4.3 below). This result leads us to consider multiplier sequences which are rapidly decreasing but which, in general, cannot be interpolated by functions $\phi \in \mathcal{L}-\mathcal{P}(-\infty, 0)$. We also prove (see Section 5) that there are sequences for which $g_{k}(t)=\sum_{j=0}^{k}\left(\begin{array}{l}k \\ j\end{array}\right) \gamma_{j} t^{j}$ 
is a CZDS for each fixed $t>0$ (Lemma 5.3). In particular, Lemma 5.3 shows the existence of a nontrivial class of CZDS, $\left\{\gamma_{k}\right\}_{k=0}^{\infty}$, for which the following geometric result is valid. Suppose that the polynomial $f(x)=\sum_{k=0}^{n} a_{k} x^{k} / k ! \in \mathbb{R}[x], a_{n} \neq 0$, has exactly $r$ real zeros, counting multiplicities. Let $F(x, y):=\sum_{k=0}^{n} \gamma_{k} x^{k} f^{(k)}(y) / k$ ! Then the curve $F(x, y)=0$ intersects each line $y=x / s, s>0$, in at least $r$ (real) points (Theorem 5.4).

\section{Polynomials which interpolate complex zero decreasing sequences}

The main theorem of this section (see Theorem 2.13 below) characterizes the class of all polynomials which interpolate CZDS. Our proof requires several preparatory results involving both CZDS and $\lambda$-sequences. We begin with an example, which is generalized in Proposition 2.2.

Example 2.1. Consider a fixed positive integer $m$ and the sequence $T=\{h(k)\}_{k=0}^{\infty}$ where $h(x)=x(x-1)(x-2) \cdots(x-m+1)$. Then, for any polynomial $f(x)=$ $\sum_{k=0}^{n} a_{k} x^{k}, T[p(x)]=\sum_{k=0}^{n} h(k) a_{k} x^{k}=x^{m} f^{(m)}(x)$. Thus, by Rolle's theorem,

$$
Z_{c}\left(\sum_{k=0}^{n} h(k) a_{k} x^{k}\right) \leq Z_{c}\left(\sum_{k=0}^{n} a_{k} x^{k}\right)
$$

and consequently $T$ is a CZDS.

Generalizing Example 2.1, we have

Proposition 2.2. Fix a positive integer $m$, and let

$$
h(x)=x(x-1)(x-2) \cdots(x-m+1) \prod_{i=1}^{p}\left(x-b_{i}\right)
$$

where $b_{i}<m$ for each $i=1, \ldots, p$. Then the sequence $\{h(k)\}_{k=0}^{\infty}$ is a CZDS.

Proof. First, we note that for any nonnegative integer $k$,

$h(k) x^{k}=k(k-1) \cdots(k-m+1)\left[\prod_{i=1}^{p}\left(k-b_{i}\right)\right] x^{k}=x^{m}\left[\prod_{i=1}^{p}\left((k-m)-\left(b_{i}-m\right)\right)\right] \frac{d^{m}}{d x^{m}} x^{k}$.

Set $g(x)=\prod_{i=1}^{p}\left(x-\left(b_{i}-m\right)\right)$, and let $f(x)=\sum_{k=0}^{n} a_{k} x^{k}$ be any polynomial. Then, by linearity, we have

$$
\begin{aligned}
\sum_{k=0}^{n} h(k) a_{k} x^{k} & =\sum_{k=m}^{n} h(k) a_{k} x^{k} \\
& =x^{m} \sum_{k=m}^{n} g(k-m) \frac{d^{m}}{d x^{m}} a_{k} x^{k} \\
& =x^{m} \sum_{k=0}^{n-m} g(k) \frac{d^{m}}{d x^{m}} a_{k+m} x^{k+m} \\
& =x^{m} \sum_{k=0}^{n-m} g(k)(k+m) \cdots(k+1) a_{k+m} x^{k}
\end{aligned}
$$


where $\sum_{k=0}^{n-m}(k+m) \cdots(k+1) a_{k+m} x^{k}$ is the $m$-th derivative of $f$. Now $g(x)$ has only real negative zeros, so by Laguerre's theorem and Rolle's theorem

$$
\begin{aligned}
& Z_{c}\left(x^{m} \sum_{k=0}^{n-m} g(k)(k+m) \cdots(k+1) a_{k+m} x^{k}\right) \\
& \quad=Z_{c}\left(\sum_{k=0}^{n-m} g(k)(k+m) \cdots(k+1) a_{k+m} x^{k}\right) \leq Z_{c}\left(f^{(m)}\right) \leq Z_{c}(f),
\end{aligned}
$$

which proves the claim.

Remark 2.3. We remark that the assumption in Proposition 2.2 that $b_{i}<m$ for each $i=1, \ldots, p$ is necessary. Indeed, set $m=1$ and $p=1$ in Proposition 2.2, so that $h(x)=x(x-b)$. If $b>1$, then the sequence $T=\{h(k)\}_{k=0}^{\infty}$ has the form $0,1-b, 2(2-b), 3(3-b), \ldots$, and thus the terms of the sequence eventually become positive even though $1-b<0$. It follows that $T$ cannot even be a multiplier sequence. A similar claim can be made for sequences arising from polynomials of the form $x(x-$ 1) $(x-2) \cdots(x-m+1)(x-b)$ with $b>m$.

In the remainder of this section, we shall make considerable use of the gamma function $\Gamma(x)=1 /(1 / \Gamma(x))$ defined on the whole complex plane except for the nonpositive integers, and its associated functions $\Gamma(\alpha, x)=\int_{x}^{\infty} e^{-t} t^{\alpha-1} d t \quad(x>0)$, called the complementary incomplete gamma function, and the incomplete gamma function $\gamma(\alpha, x)=\Gamma(\alpha)-\Gamma(\alpha, x)$ where $\Re \alpha>0$. We note that (via analytic continuation) the latter function has the representation

$$
\gamma(\alpha, x)=\sum_{k=0}^{\infty} \frac{(-1)^{k} x^{k+\alpha}}{k !(k+\alpha)}, \quad x>0, \quad \alpha \in \mathbb{C} \backslash\{0,-1,-2,-3, \ldots\} .
$$

In the proof of Theorem 2.5, we will appeal to the following lemma.

Lemma 2.4. If $\left\{\gamma_{k}\right\}$ is a CZDS, then so is $\left\{\gamma_{k+1}\right\}$.

Proof. Write $T=\left\{\gamma_{k}\right\}_{k=0}^{\infty}$ and $T_{1}=\left\{\gamma_{k+1}\right\}_{k=0}^{\infty}$. Use the fact that $T_{1}[p(x)]=$ $T[x p(x)] / x$ to obtain the conclusion.

Theorem 2.5. Let $h(x)$ be a real polynomial of degree $n$. Suppose that $h(0) \neq 0$ and that $h(x)$ has only real zeros. If the sequence $\{h(k)\}_{k=0}^{\infty}$ is a CZDS, then all the zeros of $h$ are negative.

Proof. If $\left\{\gamma_{k}\right\}_{k=0}^{\infty}$ is a CZDS, then so is $\left\{c \gamma_{k}\right\}_{k=0}^{\infty}$ for any nonzero real number $c$. Hence, we may assume that $h$ is monic. The sequence $\{h(k)\}_{k=0}^{\infty}$ cannot alternate in sign since $h$, being a polynomial, has only finitely many zeros. Since $\{h(k)\}_{k=0}^{\infty}$ is a CZDS, it is a multiplier sequence, and hence it follows that $h(k)>0$ for $k=0,1,2, \ldots$ or $h(k)<0$ for $k=0,1,2, \ldots$ (see, for example, [3, Theorem 3.4]). Without loss of generality, we may assume that $h(k)>0$ for $k=0,1,2, \ldots$ In particular, no nonnegative integer can be a zero of $h$. Since $h(x)$ has only real zeros, we may assume that the zeros of $h(x)$ are $r_{1} \leq r_{2} \leq \cdots \leq r_{n}$. We now proceed to show that if $r_{n}>0$, then the sequence $\left\{\frac{1}{h(k)}\right\}$ fails to be a $\lambda$-sequence, and hence $\{h(k)\}_{k=0}^{\infty}$ is not a CZDS, contrary to our assumption. Suppose that $r_{n}>0$. Then, using Lemma 2.4, we may assume that $r_{n}$ lies in an interval $(2 m, 2 m+1)$ for some integer $m \geq 0$. (Indeed, suppose that $r_{n}$ lies in an interval $(2 m+1,2 m+2)$ for some integer $m \geq 0$. Then, the polynomial $h_{1}(x):=h(x+1)$ vanishes at $r_{n}-1$ where $r_{n}-1 \in(2 m, 2 m+1)$. Hence, by Lemma 2.4 , the sequence $\left\{h_{1}(k)\right\}_{k=0}^{\infty}$ is also a CZDS.) 
We begin by assuming that the zeros of $h$ are simple. Since $h$ is monic, the partial fraction decomposition of $\frac{1}{h(x)}$ is of the form

$$
\frac{1}{h(x)}=\frac{1}{\prod_{i=1}^{n}\left(x-r_{i}\right)}=\sum_{i=1}^{n} \frac{A_{i}}{x-r_{i}},
$$

where $A_{i}=\left[\prod_{j \neq i}\left(r_{i}-r_{j}\right)\right]^{-1}$. Note, in particular, that $A_{n}>0$. Applying the sequence $\left\{\frac{1}{h(k)}\right\}_{k=0}^{\infty}$ to the positive function $e^{-x}$ yields

$$
F(x)=\sum_{k=0}^{\infty} \frac{(-1)^{k} x^{k}}{k ! h(k)}=\sum_{i=1}^{n} \frac{A_{i} \gamma\left(-r_{i}, x\right)}{x^{-r_{i}}}
$$

where we have used the representation (2.3) for $\gamma$. Since $r_{j} \notin\{0,1,2, \ldots\}$ and

$$
\Gamma\left(-r_{i}, x\right)=\Gamma\left(-r_{i}\right)-\sum_{k=0}^{\infty} \frac{(-1)^{k} x^{k-r_{i}}}{k !\left(k-r_{i}\right)}
$$

for $i=1, \ldots, n$, it follows that

$$
\gamma\left(-r_{i}, x\right)=\Gamma\left(-r_{i}\right)+o(1) \text { as } x \rightarrow \infty
$$

Hence, by (2.4) and (2.5), we have

$$
\begin{aligned}
F(x) & =\sum_{i=1}^{n} \frac{A_{i} \Gamma\left(-r_{i}\right)}{x^{-r_{i}}}+o(1) \text { as } x \rightarrow \infty \\
& =x^{r_{n}}\left[\sum_{i=1}^{n-1} \frac{A_{i} \Gamma\left(-r_{i}\right)}{x^{r_{n}-r_{i}}}+A_{n} \Gamma\left(-r_{n}\right)\right]+o(1) \text { as } x \rightarrow \infty .
\end{aligned}
$$

Since $-r_{n} \in(-2 m-1,-2 m), m \geq 0$, and since the real entire function $\frac{1}{\Gamma(x)}$ is negative on the interval $(-2 m-1,-2 m), \Gamma\left(-r_{n}\right)<0$, we conclude from $(2.6)$ that $F(x) \rightarrow-\infty$ as $x \rightarrow \infty$. Therefore, the sequence $\left\{\frac{1}{h(k)}\right\}$ is not a $\lambda$-sequence (see Remark 1.6).

Finally, if the zeros of $h$ are not simple, then using (2.6), a limiting argument shows that $\left\{\frac{1}{h(k)}\right\}$ again is not a $\lambda$-sequence.

If a polynomial $h(x) \in \mathcal{L}$ - $\mathcal{P}$, then the sequence $\{h(k)\}_{k=0}^{\infty}$ need not be a multiplier sequence. In the following example, we find necessary and sufficient conditions for $\{h(k)\}_{k=0}^{\infty}$ to be a multiplier sequence in the special case when $h(x)$ is a quadratic polynomial whose zeros are positive. This result will be used in the proof of Proposition 2.7.

Example 2.6. Let $h(x)=(x-r)(x-s)$ where $r, s>0$. Set $a=-(r+s)$ and $b=r s$. Then necessary and sufficient conditions for $\{h(k)\}_{k=0}^{\infty}$ to be a multiplier sequence are that

$$
-1<a<0 \text { and } a+1 \geq 2 \sqrt{b} .
$$

To see this, we first note that if $q(x):=b+(1+a) x+x^{2}$, then

$$
q(x) e^{x}=\sum_{k=0}^{\infty} h(k) \frac{x^{k}}{k !} .
$$

Now, if (2.7) holds, then $q(x)$ has only real negative zeros, and thus $q(x) e^{x} \in \mathcal{L}-\mathcal{P I}$. But then it follows from (2.8) that $\{h(k)\}_{k=0}^{\infty}$ is a multiplier sequence. Conversely, if $\{h(k)\}_{k=0}^{\infty}$ is a multiplier sequence, then the transcendental characterization of multiplier sequences (see (1.4)) implies that $q(x) e^{x} \in \mathcal{L}$ - $\mathcal{P}$ I. Since $h(0)>0$ and $h(k)>0$ for 
all sufficiently large positive integers $\mathrm{k}$, we conclude that $h(k)>0$ for $k=0,1,2 \ldots$. Thus, $(1+a)>0$, and using the quadratic formula we see that (2.7) holds. In particular, we note that if $s=r$, then $\{h(k)\}_{k=0}^{\infty}$ is a multiplier sequence if and only if $0<r \leq \frac{1}{4}$.

Proposition 2.7. Let $h(x)=(x-r)(x-s)$ with $r, s>0$. Then $\{h(k)\}_{k=0}^{\infty}$ is not a CZDS.

Proof. Since a necessary condition for a sequence to be a CZDS is that it be a multiplier sequence, we may assume that $\{h(k)\}_{k=0}^{\infty}$ is a multiplier sequence. Thus, by Example 2.6 , we may write $h(x)=x^{2}+a x+b$ where $a=-(r+s), b=r s,-1<a<0$, and $\delta=\frac{1}{2} \sqrt{a^{2}-4 b}$ satisfies $0 \leq \delta<\frac{|a|}{2}$. We shall apply the sequence $\left\{\frac{1}{h(k)}\right\}_{k=0}^{\infty}$ to $e^{-x}$ and show that the resulting entire function takes on negative values (see Remark 1.6).

First, we assume that $\delta>0$. Then, for $k \geq 1$, a standard integral formula yields

$$
\frac{1}{\delta} \int_{0}^{\infty} e^{-(k+a / 2) t} \sinh (\delta t) d t=\frac{1}{k^{2}+a k+b}
$$

In addition, for each fixed $x>0$, there is a positive constant $K=K(x, a, \delta)$, such that for all $t \geq 0$, the following inequality holds

$$
0<\left(1-e^{-x e^{-t}}\right) e^{-a t / 2} e^{\delta t}<K e^{-(a / 2-\delta+1) t},
$$

where $a / 2-\delta+1>0$ since $1+a>0$ and $\delta<|a| / 2$. The application of the sequence $\left\{\frac{1}{h(k)}\right\}_{k=0}^{\infty}$ to $e^{-x}$ thus yields

$$
\begin{aligned}
F(x) & =\sum_{k=0}^{\infty} \frac{(-1)^{k} x^{k}}{k !\left(k^{2}+a k+b\right)} \\
& =\frac{1}{b}+\frac{1}{\delta} \int_{0}^{\infty} \sum_{k=1}^{\infty} \frac{(-1)^{k} x^{k}}{k !} e^{-t k} e^{-a t / 2} \sinh (\delta t) d t \\
& =\frac{1}{b}-\frac{1}{\delta} \int_{0}^{\infty}\left(1-e^{-x e^{-t}}\right) e^{-a t / 2} \sinh (\delta t) d t
\end{aligned}
$$

where we have used $(2.9),(2.10)$ (to establish the existence of the improper integral in (2.11)), and the dominated convergence theorem, to justify the interchanging of the integral with the summation. Next, we can find a number $R=R(a, \delta)>1$ such that

$$
\begin{aligned}
& \int_{0}^{\infty}\left(1-e^{-x e^{-t}}\right) e^{-a t / 2} \sinh (\delta t) d t \geq \int_{R}^{\infty}\left(1-e^{-x e^{-t}}\right) d t \\
&=\int_{0}^{x e^{-R}}\left(\frac{1-e^{-y}}{y}\right) d y \geq \int_{0}^{x e^{-R}} \frac{1}{1+y} d y=\ln \left(x e^{-R}+1\right)
\end{aligned}
$$

where the last inequality follows from the elementary inequality

$$
\frac{1-e^{-y}}{y} \geq \frac{1}{y+1}
$$

for all $y>0$. Therefore, from (2.11) and (2.12), we deduce that $\lim _{x \rightarrow \infty} F(x)=-\infty$.

To complete the proof, we consider the case when $\delta=0$, so that $h(x)=(x-r)^{2}$ where $0<r \leq \frac{1}{4}$, by Example 2.6. Since

$$
\int_{0}^{\infty} t e^{-(k-r) t} d t=\frac{1}{(k-r)^{2}}, \quad k \geq 1
$$


a calculation similar to the one used in proving (2.11) yields

$$
\begin{aligned}
F(x) & =\sum_{k=0}^{\infty} \frac{(-1)^{k} x^{k}}{k !(k-r)^{2}} \\
& =\frac{1}{r^{2}}-\int_{0}^{\infty}\left(1-e^{-x e^{-t}}\right) e^{r t} d t .
\end{aligned}
$$

Thus, mutatis mutandis, the previous argument may be used to conclude that $\lim _{x \rightarrow \infty} F(x)=-\infty$. This shows that $F(x)$ is again negative for all sufficiently large values of $x$; thus it follows that $\left\{\frac{1}{h(k)}\right\}_{k=0}^{\infty}$ is not a $\lambda$-sequence, and a fortiori $\{h(k)\}_{k=0}^{\infty}$ is not a CZDS.

In order to prove a converse of Laguerre's theorem (see part (3) of Theorem 1.4) for polynomials, we now shall do a careful analysis in the special case of irreducible quadratic polynomials, $h(x)=x^{2}+a x+b$ where $4 b-a^{2}>0$.

Lemma 2.8. Fix $\alpha=a / 2+i \tau$ where $\tau=\frac{1}{2} \sqrt{4 b-a^{2}}$ and $4 b-a^{2}>0$. Then

$$
\left|\frac{\Gamma(\alpha, x)}{x^{\alpha}}\right| \leq \frac{2 e^{-x}}{x} \text { for all } x>\max (0, a)
$$

Proof. Writing $\left(\frac{t}{x}\right)^{\alpha}=e^{\alpha \log (t / x)}=e^{(a / 2+i \tau) \log (t / x)}$, we obtain

$$
\left|\frac{\Gamma(\alpha, x)}{x^{\alpha}}\right| \leq \frac{1}{x^{a / 2}} \int_{x}^{\infty} t^{a / 2-1} e^{-t} d t=\frac{1}{x^{a / 2}} \Gamma\left(\frac{a}{2}, x\right) .
$$

Since

$$
\frac{d}{d t}\left(t^{a / 2-1} e^{-t / 2}\right)=\frac{e^{-t / 2}}{2} t^{a / 2-2}(a-2-t),
$$

the function $t^{a / 2-1} e^{-t / 2}$ is strictly decreasing for $t>\max (0, a-2)$. In particular, for $x>\max (0, a)$,

$$
\frac{1}{x^{a / 2}} \int_{x}^{\infty}\left(t^{a / 2-1} e^{-t / 2}\right) e^{-t / 2} d t \leq \frac{x^{a / 2-1} e^{-x / 2}}{x^{a / 2}} \int_{x}^{\infty} e^{-t / 2} d t=\frac{2 e^{-x}}{x}
$$

Lemma 2.9. Fix $\alpha=a / 2+i \tau$ where $\tau=\frac{1}{2} \sqrt{4 b-a^{2}}$ and $4 b-a^{2}>0$. Then the function

$$
f(x)=-\Im\left(\frac{\Gamma(\alpha)}{x^{\alpha}}\right)+\Im\left(\frac{\Gamma(\alpha, x)}{x^{\alpha}}\right), \quad x>0,
$$

changes sign infinitely often in the interval $(0, \infty)$.

Proof. A calculation shows that

$$
\begin{aligned}
-\Im\left(\frac{\Gamma(\alpha)}{x^{\alpha}}\right) & =-\Im\left(\frac{\Gamma(\alpha) x^{\bar{\alpha}}}{x^{2 \Re \alpha}}\right) \\
& =\frac{1}{x^{a / 2}}[(\Re \Gamma(\alpha)) \sin (\tau \log x)-(\Im \Gamma(\alpha)) \cos (\tau \log x)] .
\end{aligned}
$$

Since $\Gamma(\alpha) \neq 0$, we have $(\Im \Gamma(\alpha))^{2}+(\Re \Gamma(\alpha))^{2}>0$. We first consider the case when $\Im \Gamma(\alpha) \neq 0$. Now, by Lemma 2.8 ,

$$
\left|\Im\left(\frac{\Gamma(\alpha, x)}{x^{\alpha}}\right)\right| \leq\left|\frac{\Gamma(\alpha, x)}{x^{\alpha}}\right| \leq \frac{2 e^{-x}}{x},
$$


for all $x>\max (0, a)$. Since $\lim _{x \rightarrow \infty} 2 e^{-x} x^{a / 2-1}=0$, we can find a number $x_{0}>|a|$ such that

$$
2 e^{-x} x^{a / 2-1}<|\Im \Gamma(\alpha)| \quad \text { for } x>x_{0}
$$

For $x>x_{0}$, the function $\cos (\tau \log x)$ alternately takes on the values -1 and +1 infinitely many times. Hence, if $x_{1}>x_{0}$ and if $\cos \left(\tau \log x_{1}\right)= \pm 1$, then $\sin \left(\tau \log x_{1}\right)=0$. Therefore, (2.13) yields $f\left(x_{1}\right)= \pm \Im \Gamma(\alpha) / x_{1}^{a / 2}+\Im\left(\Gamma\left(\alpha, x_{1}\right) / x_{1}^{\alpha}\right)$, which, by (2.14) and (2.15), is positive or negative according to the sign of the first term. Thus, it follows that $f(x)$ changes sign infinitely often in the interval $(0, \infty)$. If $\Im \Gamma(\alpha)=0$, then $\Re \Gamma(\alpha) \neq 0$, and we can choose $x_{0}>|a|$ such that $2 e^{-x_{0}} x_{0}^{a / 2-1}<|\Re \Gamma(\alpha)|$. The function $\sin (\tau \log x)$ assumes the values -1 and +1 infinitely often, so in this case the conclusion of the lemma also holds.

Proposition 2.10. Let

$$
F(x, a, b)=\sum_{k=0}^{\infty} \frac{(-1)^{k} x^{k}}{k !\left(k^{2}+a k+b\right)}
$$

where $a$ and $b$ are real numbers such that $4 b-a^{2}>0$. Then $F(x, a, b)$ changes sign infinitely often in the interval $(0, \infty)$.

Proof. Let $\alpha=a / 2+i \tau$ where $\tau=\frac{1}{2} \sqrt{4 b-a^{2}}$, so that $k^{2}+a k+b=(k+\alpha)(k+\bar{\alpha})$. Then, using (2.3), we can express $F(x, a, b), x>0$, as

$$
\begin{aligned}
F(x, a, b) & =\sum_{k=0}^{\infty} \frac{(-1)^{k} x^{k}}{k !\left(k^{2}+a k+b\right)} \\
& =\frac{1}{\bar{\alpha}-\alpha} \sum_{k=0}^{\infty} \frac{(-1)^{k} x^{k}}{k !}\left[\frac{1}{k+\alpha}-\frac{1}{k+\bar{\alpha}}\right] \\
& =-\frac{1}{\tau} \Im\left(\frac{\gamma(\alpha, x)}{x^{\alpha}}\right) \\
& =-\frac{1}{\tau} \Im\left(\frac{\Gamma(\alpha)}{x^{\alpha}}\right)+\frac{1}{\tau} \Im\left(\frac{\Gamma(\alpha, x)}{x^{\alpha}}\right) .
\end{aligned}
$$

Therefore, by Lemma 2.9, $F(x, a, b)$ changes sign infinitely often in the interval $(0, \infty)$.

Theorem 2.11. Let $h(x)=x^{2}+a x+b$ where $a, b \in \mathbb{R}$. Then the sequence $T=$ $\{h(k)\}_{k=0}^{\infty}$ is a CZDS if and only if either both roots of $h$ are nonpositive or one root is 0 and the other is in the interval $[0,1]$.

Proof. Suppose that $T$ is a CZDS. We will first demonstrate that the roots of $h$ must be real. To this end, we set $\Lambda=\left\{\frac{1}{h(k)}\right\}_{k=0}^{\infty}$. Now if the roots of $h$ are not real, then by Proposition 2.10, the function $\Lambda\left[e^{-x}\right]=F(x, a, b)$ changes sign infinitely often in the interval $(0, \infty)$. But then $\Lambda$ is not a $\lambda$-sequence, so $T$ is not a CZDS. This contradiction shows that the roots, call them $r$ and $s$, of $h$ must be real. By Proposition 2.7, $r$ and $s$ cannot both be positive. So suppose that $r \leq 0$ and $s \geq 0$. If $r=0$, then Remark 2.3 shows that $s$ is in the interval $[0,1]$. Therefore, we conclude that either both roots of $h$ are nonpositive, or one root is 0 , and the other is in the interval [0,1]. Since the converse implication is a direct consequence of Proposition 2.2 and Laguerre's theorem, the proof of the theorem is complete. 
Theorem 2.12. Let $h(x)$ be a real polynomial. If the sequence $T=\{h(k)\}_{k=0}^{\infty}$ is a CZDS, then all the zeros of $h$ are real.

Proof. Assume the contrary so that $h(x)$ can be expressed in the form $h(x)=\tilde{p}(x)\left(x^{2}+\right.$ $a x+b)$ where $4 b-a^{2}>0$. Then the polynomial $\tilde{p}(x)$ gives rise to the entire function $\sum_{k=0}^{\infty} \tilde{p}(k)(-1)^{k} x^{k} / k !=p(x) e^{-x}$ where $p(x)$ is a polynomial. We next approximate the entire function $p(x) e^{-x}$ by means of the polynomials $q_{n}(x)=p(x)\left[(1-x / 2 n)^{2 n}+\epsilon_{n}\right]$ where $\epsilon_{n}>0$ and $\lim _{n \rightarrow \infty} \epsilon_{n}=0$. We note, in particular, that $q_{n}(x)$ has the same number of real zeros as $p(x)$ has. Moreover, as $n \rightarrow \infty, q_{n}(x) \rightarrow p(x) e^{-x}$ uniformly on compact subsets of $\mathbb{C}$. If we set $\Lambda=\left\{\frac{1}{h(k)}\right\}_{k=0}^{\infty}$, then by Proposition 2.10 , the function

$$
\Lambda\left[p(x) e^{-x}\right]=F(x, a, b)=\sum_{k=0}^{\infty} \frac{(-1)^{k} x^{k}}{k !\left(k^{2}+a k+b\right)}
$$

has infinitely many sign changes in the interval $(0, \infty)$. Also, as $n \rightarrow \infty, f_{n}(x):=$ $\Lambda\left[q_{n}(x)\right] \rightarrow F(x, a, b)$ uniformly on compact subsets of $\mathbb{C}$. Thus, for sufficiently large $n$, each of the approximating polynomials $f_{n}(x)$ has more real zeros than $p(x)$ has. Since $T$ is a CZDS, $Z_{c}\left(T\left[f_{n}(x)\right]\right) \leq Z_{c}\left(f_{n}(x)\right)$, and consequently, for $n$ sufficiently large, the polynomial $T\left[f_{n}(x)\right]=T\left[\Lambda\left[q_{n}(x)\right]\right]=q_{n}(x)$ has more real zeros than $p(x)$ has. This is the required contradiction, and thus the proof of the theorem is complete.

Finally, to summarize the foregoing results, we state

Theorem 2.13. Let $h(x)$ be a real polynomial. The sequence $T=\{h(k)\}_{k=0}^{\infty}$ is a complex zero decreasing sequence (CZDS) if and only if either

(1) $h(0) \neq 0$ and all the zeros of $h$ are real and negative, or

(2) $h(0)=0$ and the polynomial $h(x)$ has the form given by (2.2) in Proposition 2.2.

Proof. Suppose that $T$ is a CZDS. Then case (1) is a consequence of Theorems 2.12 and 2.5. In case $(2)$, set $h(x)=x(x-1)(x-2) \cdots(x-m+1) \prod_{i=1}^{p}\left(x-b_{i}\right)$, and let $g(x)=h(x+m)$. Then, by Lemma 2.4 , the sequence $\{g(k)\}_{k=0}^{\infty}$ is also a CZDS. Since $g(0) \neq 0$, by case (1), all the zeros of the polynomial $g$ are real and negative, and hence we see that $b_{i}-m<0$, or $b_{i}<m$, for $i=1,2, \ldots, p$. Conversely, if $h(0) \neq 0$ and all the zeros of $h$ are real and negative, then $T$ is a CZDS by Laguerre's theorem (see part(3) of Theorem 1.4). If $h(0)=0$ and the polynomial $h(x)$ has the form given by (2.2), then $T$ is a CZDS by Proposition 2.2 .

\section{Some extensions to transcendental entire functions}

It was noted in the Introduction (see the comment following Theorem 1.4) that Theorem 2.13 is not true in general if the polynomial $h(x)$ is replaced with a transcendental entire function. On the other hand, we know that sequences generated by entire functions which are limits of polynomials satisfying Laguerre's theorem (see part (3) of Theorem 1.4) again give rise to complex zero decreasing sequences. The sequence $\left\{\frac{1}{k !}\right\}_{k=0}^{\infty}$ is one of the classical paradigms of CZDS (see, for example, [20, p. 14]) which arises from the reciprocal of the gamma function. In this section, we shall establish some limited generalizations of the results of the previous section in the case of transcendental entire functions. The main emphasis will be on sequences with only finitely many nonzero terms. Sequences that end in a string of zeros are in a certain sense complementary to those which begin with zeros; in particular, compare Proposition 2.2 and Corollary 3.3, and note the restrictions imposed on the positive zeros of the 
interpolating functions involved. In addition, Corollary 3.3 provides an extension of part (2) of Theorem 1.4.

Theorem 3.1. For $n, r \geq 0$, the sequence $\left\{\left(\begin{array}{c}n \\ k-r\end{array}\right)\right\}_{k=0}^{\infty}$ is a CZDS where, by convention, $\left(\begin{array}{l}n \\ k\end{array}\right)=0$ if $k>n$ or $k<0$.

Proof. To an arbitrary polynomial $p(x)=\sum_{k=0}^{m} a_{k} x^{k}$ we apply a series of manipulations, each of which leaves the number of nonreal zeros unchanged or reduced, ending with the desired application of the binomial sequence to $p(x)$.

We first consider the case when $m \geq n$ and $r=0$. (1) We begin by reversing the coefficients of $p(x)$, forming $x^{m} p\left(x^{-1}\right)=\sum_{k=0}^{m} a_{m-k} x^{k}$. (2) Next, we apply the operator $x^{m-n} d^{m-n} / d x^{m-n}$, resulting in the polynomial

$$
\sum_{k=m-n}^{m} k(k-1) \cdots(k-m+n+1) a_{m-k} x^{k} .
$$

(3) To this we apply the sequence $\left\{\frac{1}{k !}\right\}_{k=0}^{\infty}$, which yields $\sum_{k=m-n}^{m} a_{m-k} x^{k} /(k-m+n)$ !. (4) Division by $x^{m-n}$ results in $q(x)=\sum_{k=0}^{n} a_{n-k} x^{k} / k$ !. (5) Reversing the coefficients of $q(x)$ gives us $x^{n} q\left(x^{-1}\right)=\sum_{k=0}^{n} a_{k} x^{k} /(n-k) !$. (6) Another application of the sequence $\left\{\frac{1}{k !}\right\}_{k=0}^{\infty}$ results in $\sum_{k=0}^{n} a_{k} x^{k} / k !(n-k)$ !. (7) Finally, multiplication by $n$ ! yields the desired polynomial $\sum_{k=0}^{n}\left(\begin{array}{l}n \\ k\end{array}\right) a_{k} x^{k}$.

The second case, $m<n, r=0$, is very similar. Change step (2) to multiplication by $x^{n-m}$ and step (4) to division by $x^{n-m}$, so that the final result is $\sum_{k=0}^{m}\left(\begin{array}{l}n \\ k\end{array}\right) a_{k} x^{k}$, as desired.

Finally, if $r>0$, first form $\sum_{k=0}^{m}\left(\begin{array}{c}n+r \\ k\end{array}\right) a_{k} x^{k}$ using the preceding cases. Differentiate $r$ times, divide by $(n+r)(n+r+1) \cdots(n+1)$, and then multiply by $x^{r}$ to obtain $\sum_{k=0}^{m}\left(\begin{array}{c}n \\ k-r\end{array}\right) a_{k} x^{k}$, as required.

Corollary 3.2. Let $n$ be a positive integer. If $\left\{\gamma_{k}\right\}_{k=0}^{\infty}$ is a CZDS, then so is

$$
\left\{\frac{\gamma_{0}}{n !}, \frac{\gamma_{1}}{(n-1) !}, \frac{\gamma_{2}}{(n-2) !}, \ldots, \frac{\gamma_{n}}{0 !}, 0,0,0, \ldots\right\} \text {. }
$$

Proof. This is proved by following the steps in the proof of Theorem 3.1 and using the sequence $\left\{\gamma_{k}\right\}_{k=0}^{\infty}$ in place of $\left\{\frac{1}{k !}\right\}_{k=0}^{\infty}$ in step (6).

Corollary 3.3. Let $h(z) \in \mathcal{L}$-P. If $h(z)$ has no zeros in $[0, n]$ and if $h(z)$ has a zero at every integer greater than $n$, then $\{h(k)\}_{k=0}^{\infty}$ is a CZDS.

Proof. Let $h(z) \in \mathcal{L}$-P . Suppose that $h(z)$ has no zeros in $[0, n]$, and suppose that $h(z)$ has a zero at every integer greater than $n$. Set $g(z)=1 / \Gamma(n+1-z)$, so that $g(z)$ satisfies the same hypotheses as $h(z)$. By Corollary 3.2 , with $\gamma_{k}=1$ for all $k,\{g(k)\}_{k=0}^{\infty}$ is a CZDS. Then elementary considerations involving removable singularities show that the function $\phi(z)=h(z) / g(z)=h(z) \Gamma(n+1-z)$ is an entire function. Moreover, it is easy to see that $\phi \in \mathcal{L}$ - $\mathcal{P}$, and none of the zeros of $\phi$ lie in the interval $(0, n)$. Therefore, by Laguerre's theorem (see part(2) of Theorem 1.4), for any real polynomial $f(x)=\sum_{k=0}^{m} a_{k} x^{k}$ where $\operatorname{deg} f=m \leq n$,

$$
Z_{c}\left(\sum_{k=0}^{m} \phi(k) a_{k} x^{k}\right) \leq Z_{c}(f(x)) .
$$


Now if $\operatorname{deg} f=m>n$, then another application of Laguerre's theorem and the fact that the sequence $\{g(k)\}_{k=0}^{\infty}$ is a CZDS shows that

$$
\begin{aligned}
Z_{c}\left(\sum_{k=0}^{m} h(k) a_{k} x^{k}\right) & =Z_{c}\left(\sum_{k=0}^{m} \phi(k) g(k) a_{k} x^{k}\right) \\
& =Z_{c}\left(\sum_{k=0}^{n} \phi(k) g(k) a_{k} x^{k}\right) \\
& \leq Z_{c}\left(\sum_{k=0}^{n} g(k) a_{k} x^{k}\right) \\
& =Z_{c}\left(\sum_{k=0}^{m} g(k) a_{k} x^{k}\right) \\
& \leq Z_{c}(f(x)) .
\end{aligned}
$$

Therefore, the sequence $\{h(k)\}_{k=0}^{\infty}$ is a CZDS.

A multiplier sequence $\left\{\gamma_{k}\right\}_{k=0}^{\infty}$ is a CZDS if and only if the sequence $\left\{c r^{k} \gamma_{k}\right\}_{k=0}^{\infty}$ where $c$ and $r$ are nonzero real numbers is a CZDS. Indeed, we simply note that if $p(x)$ is a polynomial and $T=\left\{c r^{k}\right\}_{k=0}^{\infty}$, then $T[p(x)]=c p(r x)$. Of greater importance to us is the fact that a multiplier sequence can be interpolated (by a function in $\mathcal{L}-\mathcal{P}$ with zeros restricted to certain intervals) if and only if the scaled sequence can be so interpolated. For future reference, we record a precise version of this fact, the proof of which is clear.

Lemma 3.4. If the sequence $\left\{\gamma_{k}\right\}$ is interpolated by a function $\phi(x)$, then, for any $r>0$, the sequence $\left\{\gamma_{k} r^{k}\right\}$ is interpolated by $\phi(x) e^{x \ln r}$.

It is not known if all CZDS with only finitely many nonzero terms arise from a Laguerre interpolation, that is, one in which the interpolating function satisfies the hypotheses of Laguerre's theorem. However, as a partial converse to Corollary 3.3, we consider sequences with at most three nonzero terms.

Proposition 3.5. (1) A sequence of the form $\{a, b, 0,0, \ldots\}$ with $a, b>0$ is always a CZDS. All such sequences can be interpolated by functions in $\mathcal{L}-\mathcal{P}$ with zeros outside $[0,1]$.

(2) A sequence of the form $\{a, b, c, 0,0, \ldots\}$ with $a, b, c>0$ is a CZDS if and only if $b^{2}-2 a c \geq 0$. All such sequences can be interpolated by functions in $\mathcal{L}-\mathcal{P}$ with zeros outside $[0,2]$.

Proof. (1) The application of the sequence to any polynomial yields a polynomial of degree one, so the sequence must be a CZDS. A function of the proper form which interpolates the sequence is $((b-a) x+a) / \Gamma(2-x)$, as is easily checked since $\Gamma(2-x)$ equals 1 at zero and one, and equals 0 at all integers greater than one.

(2) The condition for $\{a, b, c, 0,0, \ldots\}$ to be a multiplier sequence is that $a+b x+$ $c x^{2} / 2$ ! have only real negative zeros [3]. Since applying this sequence to any polynomial results in a polynomial of degree at most two, this also is equivalent to being a CZDS. The condition given is the usual discriminant condition for real zeros.

By Lemma 3.4, we may scale the sequence with the constant $r=\sqrt{2 a / c}$. We also may multiply by a constant, in this case $(2 a)^{-1}$, resulting in the normalized sequence $\left\{\frac{1}{2}, d, 1,0,0, \ldots\right\}$ to be interpolated, where $d \geq 1$. In order to get the zeros in the sequence, we use a function of the form $p(x) / \Gamma(3-x)$ where $p(x)$ is a polynomial. 
Since $1 / \Gamma(3-k), k=0,1, \ldots$, takes the values $\frac{1}{2}, 1,1,0,0, \ldots$, the function $p(x)$ must satisfy $p(0)=1, p(1)=d$, and $p(2)=1$. If $d=1$, we can take $p(x)=1$. Otherwise, the unique quadratic with this property is $p(x)=(1-d) x^{2}+(2 d-2) x+1$, with zeros $x=1 \pm \sqrt{d^{2}-d} /(d-1)$ which lie outside $[0,2]$ for any $d>1$.

Remark 3.6. The interpolation problem for a sequence with four nonzero terms is much more complicated. It is not hard to find a CZDS which cannot be interpolated by a function of the form $p(x) / \Gamma(4-x)$ where $p(x)$ is a polynomial of degree 3 all of whose zeros are real and lie outside the interval $[0,3]$. In this case, the sequence $\{a, b, c, d, 0,0, \ldots\}$, with $a, b, c, d>0$, is a CZDS if and only if it is a multiplier sequence. This occurs if and only if $a+b x+c x^{2} / 2 !+d x^{3} / 3$ ! only has real negative zeros, which is equivalent to $-9 a^{2} d^{2}+18 a b c d-8 b^{3} d-6 a c^{3}+3 b^{2} c^{2} \geq 0$. As a concrete example, consider the CZDS $\{32,24,12,3,0,0, \ldots\}$. The interpolating polynomial $p$ must satisfy $p(0)=192, p(1)=48, p(2)=12, p(4)=3$ because of the denominator $\Gamma(4-x)$. If $p$ has degree 3 , this polynomial is determined by the conditions and has two of its zeros between 1 and 2 .

Proposition 3.7. Let $p(x)$ be a polynomial, $p(x) e^{\sigma x}=\sum_{0}^{\infty} \gamma_{k} x^{k} / k$ !, where $\sigma>0$ and $p(0) \neq 0$. The sequence $\left\{\gamma_{k}\right\}_{k=0}^{\infty}$ is a CZDS if and only if there exists a polynomial $h(x) \in \mathcal{L}-\mathcal{P}(-\infty, 0)$ such that $h(x) e^{x \ln \sigma}$ interpolates $\left\{\gamma_{k}\right\}_{k=0}^{\infty}$.

Proof. By Laguerre's theorem, the existence of such a polynomial implies that $\left\{\gamma_{k}\right\}_{k=0}^{\infty}$ is a CZDS. For the converse, we write $p(x)=\sum_{j=0}^{m} b_{j} x^{j} / j$ ! and

$$
p\left(\frac{x}{\sigma}\right) e^{x}=\sum_{k=0}^{\infty} \frac{\gamma_{k}}{\sigma^{k}} \frac{x^{k}}{k !}=\sum_{k=0}^{\infty} \frac{g_{k}^{*}(\sigma)}{\sigma^{k}} \frac{x^{k}}{k !}
$$

where

$$
p(x) e^{\sigma x}=\sum_{k=0}^{\infty} g_{k}^{*}(\sigma) \frac{x^{k}}{k !}
$$

and

$$
g_{k}^{*}(\sigma)=\sum_{j=0}^{\min (k, m)}\left(\begin{array}{l}
k \\
j
\end{array}\right) b_{j} \sigma^{k-j}=\sigma^{k} \sum_{j=0}^{\min (k, m)}\left(\begin{array}{l}
k \\
j
\end{array}\right) \frac{b_{j}}{\sigma^{j}}
$$

(see [9]). Let $h(x)$ be the polynomial $\sum_{j=0}^{m} b_{j}\left(\begin{array}{l}x \\ j\end{array}\right) / \sigma^{j}$. Now $\left\{\gamma_{k}\right\}_{k=0}^{\infty}$ is a CZDS if and only if $\left\{\gamma_{k} / \sigma^{k}\right\}_{k=0}^{\infty}$ is a CZDS, so $p\left(\frac{x}{\sigma}\right) e^{x}=\sum_{k=0}^{\infty} h(k) x^{k} / k$ ! implies by Theorem 2.13 that $h(x)$ has only real negative zeros. Since $\gamma_{k}=h(k) \sigma^{k}$, the conclusion follows.

Remark 3.8. Since multiplier sequences arise as sequences of Taylor coefficients, $\gamma_{k}$, of functions of the form $e^{\sigma x} \prod_{1}^{\infty}\left(1+x / x_{k}\right)$ (as opposed to having only finitely many zeros), it would be desirable to be able to prove that if $\left\{\gamma_{k}\right\}_{k=0}^{\infty}$ is a CZDS, then so are the sequences obtained from approximating functions $\phi_{n}(x)=e^{\sigma x} \prod_{1}^{n}\left(1+x / x_{k}\right)$. In fact, removing even one zero causes trouble. For example, let $a, b, c>0$ and assume that the coefficients of $(x+a)(x+b)(x+c) e^{x}$ form a CZDS. By Theorem 2.13, we know that this is equivalent to requiring the polynomial $x^{3}+(c+b+a-3) x^{2}+((b+$ $a-1) c+(a-1) b-a+2) x+a b c$ to have only real negative zeros. For example, if $a=1$ and $b=9$, this is (approximately) equivalent to $c<0.00433$ or $3.5197<c<4.1133$ or $c>15.963$. Choosing $c$ sufficiently close to any of the endpoints of these intervals will yield a function in which deleting an appropriate factor, either $x+a$ or $x+b$, will give rise to a sequence which is not a CZDS. The reason for this is that the Taylor coefficients of $(x+r)(x+s) e^{x}, 0<r<s$, form a CZDS if and only if $1+r+2 \sqrt{r} \leq s$, as one can check by using Theorem 2.13 . 


\section{A generalization of Hutchinson's theorem and some classes of zero decreasing sequences}

If $T=\left\{\gamma_{k}\right\}_{k=0}^{\infty}$ is a CZDS, then for each fixed $t>0$, the sequence $\left\{g_{k}(t)\right\}_{k=0}^{\infty}$, where $g_{k}(t)=\sum_{j=0}^{k}\left(\begin{array}{l}k \\ j\end{array}\right) \gamma_{j} t^{j}$, is a multiplier sequence because

$$
e^{x} \sum_{k=0}^{\infty} \frac{\gamma_{k}}{k !} x^{k} t^{k}=\sum_{k=0}^{\infty} g_{k}(t) \frac{x^{k}}{k !} \in \mathcal{L}-\mathcal{P} \mathrm{I} .
$$

In general, the multiplier sequence $\left\{g_{k}(t)\right\}_{k=0}^{\infty}$ need not be a CZDS for all $t>0$. Indeed, consider

$$
(1+x)^{2} e^{x}=\sum_{k=0}^{\infty} \frac{1+k+k^{2}}{k !} x^{k} \in \mathcal{L}-\mathcal{P I},
$$

for which the sequence $\left\{\gamma_{k}\right\}_{k=0}^{\infty}=\{1,2,2,0,0, \ldots\}$ is a CZDS by Proposition 3.5. If we set $h(x)=1+x+x^{2}$, then $g_{k}(1)=h(k)$, and by Theorem $2.13,\{h(k)\}_{k=0}^{\infty}=\left\{g_{k}(1)\right\}_{k=0}^{\infty}$ is not a CZDS. (Also see Example 1.8.) In this section, we will establish the existence of a class of CZDS $\left\{\gamma_{k}\right\}_{k=0}^{\infty}$ for which the sequence $\left\{g_{k}(t)\right\}_{k=0}^{\infty}$ is a CZDS for all $t \geq \gamma_{1}$ where $g_{k}(t)=\sum_{j=0}^{k}\left(\begin{array}{c}k \\ j\end{array}\right) \gamma_{j} t^{j}$ (see Corollary 4.7). To this end, we first generalize a classical theorem of Hutchinson [12] (also see Hardy [10] or [11, pp. 95-99], Petrovitch [21], and the recent paper by Kurtz [17, p. 259]).

Theorem 4.1. (Whittaker [28, p. 53]) Let $\left\{d_{k}\right\}_{k=0}^{\infty}$ be a sequence of complex numbers such that $\varlimsup_{k \rightarrow \infty}\left|d_{k}\right|^{1 / k}=L<1$. Then the series

$$
f(z)=\sum_{n=0}^{\infty} d_{n}\left(\begin{array}{l}
z \\
n
\end{array}\right)=\sum_{n=0}^{\infty} d_{n} \frac{z(z-1) \cdots(z-n+1)}{n !}
$$

converges uniformly on compact subsets of $\mathbb{C}$, to the entire function $f(z)$. Moreover, $f(z)$ satisfies $\varlimsup_{r \rightarrow \infty} \log M(r, f) / r \leq \log \frac{1}{1-L}$ where $M(r, f)=\max _{|z|=r}|f(z)|$.

Lemma 4.2. Let $\left\{\gamma_{k}\right\}_{k=0}^{\infty}$ be a sequence of positive real numbers. If $\alpha>0$ and if the Turán inequalities $\gamma_{n}^{2} \geq \alpha^{2} \gamma_{n-1} \gamma_{n+1}$ hold for $n \geq 1$, then $\gamma_{n} \leq\left(\gamma_{0} / \alpha^{n(n-1) / 2}\right)\left(\gamma_{1} / \gamma_{0}\right)^{n}$ for $n \geq 0$. Moreover, if $\alpha>1$, then $f(z)=\sum_{n=0}^{\infty} \gamma_{n}\left(\begin{array}{l}z \\ n\end{array}\right)$ represents an entire function of order $\rho(f)=\varlimsup_{r \rightarrow \infty} \log \log M(r, f) / \log r=0$.

Proof. The first conclusion is a consequence of $\gamma_{n}^{2} \geq \alpha^{2} \gamma_{n-1} \gamma_{n+1}$ and an elementary induction argument. If $\alpha>1$, it implies that $\varlimsup_{n \rightarrow \infty} \gamma_{n}^{1 / n}=0$, so we see that $f(z)$ is an entire function by Theorem 4.1. To check the order of the entire function $f(z)$, we use the following estimates for $|z| \geq 1$ :

$$
\begin{aligned}
|f(z)| & \leq \sum_{n=0}^{\infty} \frac{\gamma_{n}|z|^{n}}{n !}\left(1+\frac{1}{|z|}\right)\left(1+\frac{2}{|z|}\right) \cdots\left(1+\frac{n-1}{|z|}\right) \\
& \leq \sum_{n=0}^{\infty} \gamma_{n}|z|^{n} \\
& \leq \gamma_{0} \sum_{n=0}^{\infty} \frac{1}{\alpha^{n(n-1) / 2}}\left(\frac{\gamma_{1}}{\gamma_{0}}|z|\right)^{n} \\
& =\gamma_{0} M(F, r) \quad(|z|=r)
\end{aligned}
$$

where $F(z)=\sum_{n=0}^{\infty} a_{n} z^{n}$ and $a_{n}=\left(1 / \alpha^{n(n-1) / 2}\right)\left(\gamma_{1} / \gamma_{0}\right)^{n}$. Thus $M(r, f) \leq M(r, F)$ for $r \geq 1$. But $F(z)$ is of order zero since $\rho(F)=\varlimsup_{n \rightarrow \infty}-n \log n / \log \left|a_{n}\right|=0$, see 
$[1$, p. 9]. Thus,

$$
\rho(f)=\varlimsup_{r \rightarrow \infty} \frac{\log \log M(r, f)}{\log r} \leq \varlimsup_{r \rightarrow \infty} \frac{\log \log M(r, F)}{\log r}=\rho(F)=0 .
$$

Theorem 4.3. Let $\phi(x)=\sum_{n=0}^{N} \frac{\gamma_{n}}{n !} x^{n}$, with $\gamma_{0}=1, \gamma_{n}>0$, and suppose that the Turán inequalities, $\gamma_{n}^{2} \geq \alpha^{2} \gamma_{n-1} \gamma_{n+1}$, hold for $n=1,2, \ldots, N-1$ where

$$
\alpha:=\max \left(2, \frac{\sqrt{2}}{2}\left(1+\sqrt{1+\gamma_{1}}\right)\right) \text {. }
$$

Then the polynomial $\tilde{\phi}(x)=\sum_{n=0}^{N} \gamma_{n}\left(\begin{array}{l}x \\ n\end{array}\right)$ has only real, simple negative zeros.

Proof. Define the positive numbers $b_{k}, k=1, \ldots, N$, recursively by $b_{0}=1$ and the formulas

$$
\frac{\gamma_{1}}{1 !}=\frac{1}{b_{1}}, \quad \frac{\gamma_{2}}{2 !}=\frac{1}{b_{1} b_{2}}, \quad \cdots, \frac{\gamma_{N}}{N !}=\frac{1}{b_{1} b_{2} \cdots b_{N}} .
$$

The Turán inequalities assumed for the numbers $\gamma_{n}$ imply that

$$
b_{n+1} \geq \alpha^{2}\left(1+\frac{1}{n}\right) b_{n}, \quad n=1,2, \ldots, N-1,
$$

where $\alpha$ is defined by (4.1). Let

$$
x_{k}=-\sqrt{b_{2 k-1} b_{2 k}}, \quad k=1,2, \ldots, \quad r=\left\lfloor\frac{N}{2}\right\rfloor
$$

where $\lfloor x\rfloor$ denotes the floor function, and

$$
y_{k}=-\sqrt{b_{2 k} b_{2 k+1}}, \quad k=1,2, \ldots, \quad s=\left\lfloor\frac{N-1}{2}\right\rfloor .
$$

For $x \neq 0$, we set

$$
t_{n}(x)=\gamma_{n}\left(\begin{array}{l}
x \\
n
\end{array}\right)=\frac{x^{n}}{b_{1} \cdots b_{n}} \pi_{n}(x), \quad n=0,1, \ldots, N
$$

where $\pi_{0}(x)=1$ and $\pi_{n}(x)=\left(1-\frac{1}{x}\right)\left(1-\frac{2}{x}\right) \cdots\left(1-\frac{n-1}{x}\right)$. Then $t_{n}(x)$ satisfies the recursion formula

$$
t_{0}(x)=1, \quad t_{n}(x)=t_{n-1}(x) \frac{x}{b_{n}}\left(1-\frac{n-1}{x}\right), \quad n=1, \ldots, N .
$$

Evaluating (4.5) at $x_{k}$, which is negative, we obtain

$$
t_{n}\left(x_{k}\right) t_{n-1}\left(x_{k}\right)<0, \quad k=1, \ldots, r, \quad n=1, \ldots, N .
$$

Now, for $1 \leq n \leq 2 k-1$, we have

$$
\frac{\left|x_{k}\right|}{b_{n}}\left(1-\frac{n-1}{x_{k}}\right)=\frac{\left|x_{k}\right|}{b_{n}}\left(1+\frac{n-1}{\left|x_{k}\right|}\right) \geq \frac{\left|x_{k}\right|}{b_{n}}=\sqrt{\frac{b_{2 k-1} b_{2 k}}{b_{n}^{2}}} \geq 1
$$

by (4.2). Combining this with (4.5) yields

$$
\left|t_{n}\left(x_{k}\right)\right| \geq\left|t_{n-1}\left(x_{k}\right)\right|, \quad n=1,2, \ldots, 2 k-1 .
$$


Now consider

$$
\begin{aligned}
T_{k} & :=t_{2 k-2}\left(x_{k}\right)+t_{2 k-1}\left(x_{k}\right)+t_{2 k}\left(x_{k}\right) \\
& =\frac{x_{k}^{2 k-2}}{b_{1} \cdots b_{2 k-2}} \pi_{2 k-2}\left(x_{k}\right)+\frac{x_{k}^{2 k-1}}{b_{1} \cdots b_{2 k-1}} \pi_{2 k-1}\left(x_{k}\right)+\frac{x_{k}^{2 k}}{b_{1} \cdots b_{2 k}} \pi_{2 k}\left(x_{k}\right) \\
& =C_{k}\left\{1+\frac{x_{k}}{b_{2 k-1}}\left(1+\frac{2 k-2}{\left|x_{k}\right|}\right)+\frac{x_{k}^{2}}{b_{2 k-1} b_{2 k}}\left(1+\frac{2 k-2}{\left|x_{k}\right|}\right)\left(1+\frac{2 k-1}{\left|x_{k}\right|}\right)\right\} \\
& =C_{k}\left\{1-\sqrt{\frac{b_{2 k}}{b_{2 k-1}}}\left(1+\frac{2 k-2}{\left|x_{k}\right|}\right)+\left(1+\frac{2 k-2}{\left|x_{k}\right|}\right)\left(1+\frac{2 k-1}{\left|x_{k}\right|}\right)\right\}
\end{aligned}
$$

where

$$
C_{k}=\frac{x_{k}^{2 k-2}}{b_{1} \cdots b_{2 k-2}}\left(1-\frac{1}{x_{k}}\right)\left(1-\frac{2}{x_{k}}\right) \cdots\left(1-\frac{2 k-3}{x_{k}}\right)
$$

for $k>1$, and $C_{1}=1$. Our immediate goal is to show that $T_{k}<0$. Since $C_{k}>0$, we have $T_{k}<0$ if

$$
\sqrt{\frac{b_{2 k}}{b_{2 k-1}}}>\left(1+\frac{2 k-2}{\left|x_{k}\right|}\right)^{-1}+\left(1+\frac{2 k-1}{\left|x_{k}\right|}\right) .
$$

From (4.2), we obtain

$$
\sqrt{\frac{b_{2 k}}{b_{2 k-1}}} \geq \alpha \sqrt{1+\frac{1}{2 k-1}}
$$

so it will suffice to show that

$$
\alpha \geq\left(1+\frac{2 k-2}{\left|x_{k}\right|}\right)^{-1}\left(1+\frac{1}{2 k-1}\right)^{-1 / 2}+\left(1+\frac{2 k-1}{\left|x_{k}\right|}\right)\left(1+\frac{1}{2 k-1}\right)^{-1 / 2}
$$

From (4.2), (4.3), and $b_{1}=1 / \gamma_{1}$ we obtain

$$
\left|x_{k}\right| \geq \frac{\alpha^{4 k-3} \sqrt{2 k(2 k-1)}}{\gamma_{1}} .
$$

In the last term of (4.9), we replace $\left|x_{k}\right|$ by this lower bound and replace $1+\frac{2 k-2}{\left|x_{k}\right|}$ by a lower bound of 1 to obtain

$$
\alpha^{4 k-2}-\sqrt{2\left(2-\frac{1}{k}\right)} \alpha^{4 k-3}-\left(1-\frac{1}{2 k}\right) \gamma_{1} \geq 0 .
$$

For any fixed $\gamma_{1}>0$, one can easily check that the positive root of the polynomial in (4.11) approaches 2 from below as $k \rightarrow \infty$. Thus, we require $\alpha \geq 2$. Also, for each fixed $\gamma_{1}>0$ and $\alpha \geq 2$, we have $\alpha^{4 k-2}-\sqrt{2(2-1 / k)} \alpha^{4 k-3}-(1-1 / 2 k) \gamma_{1} \geq \alpha^{2}-\sqrt{2} \alpha-\frac{\gamma_{1}}{2}$ for each $k=1,2, \ldots$, so the maximum condition on $\alpha$ is obtained when $k=1$, yielding $\alpha \geq \frac{\sqrt{2}}{2}\left(1+\sqrt{1+\gamma_{1}}\right)$. In all cases, $\alpha \geq \max \left(2, \frac{\sqrt{2}}{2}\left(1+\sqrt{1+\gamma_{1}}\right)\right)$ suffices to guarantee that (4.9) holds and hence that $T_{k}<0$.

Finally, we check that

$$
\left|t_{2 k+1}\left(x_{k}\right)\right| \geq\left|t_{2 k+2}\left(x_{k}\right)\right| \geq \cdots \geq\left|t_{N}\left(x_{k}\right)\right| \text {. }
$$

For $1 \leq j \leq N-1$ and $2 k+j+1 \leq\left\lfloor\frac{N}{2}\right\rfloor=r$, we simplify the following ratio, using (4.3) and (4.5),

$$
\left|\frac{t_{2 k+j}\left(x_{k}\right)}{t_{2 k+j+1}\left(x_{k}\right)}\right|^{2}=\frac{b_{2 k+j+1}^{2}}{b_{2 k-1} b_{2 k}}\left(1+\frac{2 k+j}{\left|x_{k}\right|}\right)^{-2}=\frac{b_{2 k+j+1}^{2}}{\left(\sqrt{b_{2 k-1} b_{2 k}}+2 k+j\right)^{2}} .
$$


By $(4.2), b_{2 k} / \alpha \geq \sqrt{b_{2 k-1} b_{2 k}}$, so

$$
\frac{b_{2 k+j+1}}{\sqrt{b_{2 k-1} b_{2 k}}+2 k+j} \geq \frac{b_{2 k+j+1}}{b_{2 k} / \alpha+2 k+j} .
$$

Thus, to prove (4.12), it suffices to show that $b_{2 k+j+1}-b_{2 k} / \alpha \geq 2 k+j$. Again, using $(4.2)$

$$
\begin{aligned}
b_{2 k+j+1}-\frac{b_{2 k}}{\alpha} & \geq\left(\alpha^{2 j+2}-\frac{1}{\alpha}\right) b_{2 k} \\
& \geq \frac{\left(\alpha^{2 j+2}-1 / \alpha\right) \alpha^{4 k-2}}{\gamma_{1}} \\
& =\alpha^{4 k+2 j}\left(1-\frac{1}{\alpha^{2 j+3}}\right) \frac{1}{\gamma_{1}} .
\end{aligned}
$$

Since $\alpha^{2} \geq\left(1+\sqrt{1+\gamma_{1}}\right)^{2} / 2=1+\sqrt{1+\gamma_{1}}+\gamma_{1} / 2$ and $\alpha \geq 2$, we have

$$
\alpha^{4 k+2 j}\left(1-\frac{1}{\alpha^{2 j+3}}\right) \frac{1}{\gamma_{1}} \geq \frac{31}{32}\left(1+\sqrt{1+\gamma_{1}}+\gamma_{1} / 2\right)^{2 k+j} \frac{1}{\gamma_{1}} .
$$

For $u>0$ and any positive integer $m \geq 2, \frac{31}{32}(1+\sqrt{1+u}+u / 2)^{m} \geq m u$. Therefore, it follows from (4.13), (4.14), and (4.15) that (4.12) holds.

Next, it follows from (4.6), (4.7), (4.8) (since $\left.T_{k}<0\right)$, and (4.12) that $\tilde{\phi}\left(x_{k}\right)=$ $\left(1+t_{1}\left(x_{k}\right)\right)+\left(t_{2}\left(x_{k}\right)+t_{3}\left(x_{k}\right)\right)+\cdots+\left(t_{2 k-4}\left(x_{k}\right)+t_{2 k-3}\left(x_{k}\right)\right)+T_{k}+\left(t_{2 k+1}\left(x_{k}\right)+\right.$ $\left.t_{2 k+2}\left(x_{k}\right)\right)+\cdots+t_{N}\left(x_{k}\right)<0$.

A similar argument shows that $\tilde{\phi}\left(y_{k}\right)>0, k=1, \ldots, s$, where $y_{k}$ is defined by (4.4). Thus the sequence $\tilde{\phi}(0), \tilde{\phi}\left(x_{1}\right), \tilde{\phi}\left(y_{1}\right), \tilde{\phi}\left(x_{2}\right), \ldots$, ending with $\tilde{\phi}\left(x_{r}\right)$ if $N$ is even, and with $\tilde{\phi}\left(y_{s}\right)$ if $N$ is odd, has $N-1$ sign changes. The sign of $\tilde{\phi}$ must change once more to the left of the last point $\left(x_{r}\right.$ or $\left.y_{s}\right)$, since the leading coefficient $\gamma_{N} / N$ ! of $\tilde{\phi}$ is positive. Therefore, $\tilde{\phi}$ has $N$ real, simple negative roots and the theorem is proved.

Remark 4.4. Theorem 4.3 is a generalization of a classical theorem of Hutchinson [12] in which he shows that $\phi(x)=\sum_{n=0}^{N} \gamma_{n} x^{n} / n$ ! has only real, simple negative zeros. Our result shows that $\tilde{\phi}(x)=\sum_{n=0}^{N} \gamma_{n}\left(\begin{array}{l}x \\ n\end{array}\right)$ has only real, simple negative zeros. Consequently, $\left\{\tilde{\phi}(k\}_{k=0}^{\infty}\right.$ is a multiplier sequence, whence $\sum_{k=0}^{\infty} \tilde{\phi}(k) x^{k} / k !=\phi(x) e^{x}$ has only real negative zeros, which implies Hutchinson's result.

Corollary 4.5. Let $\phi(x)=\sum_{n=0}^{\infty} \gamma_{n} x^{n} / n$ ! with $\gamma_{0}=1, \gamma_{n} \geq 0$ for $n=1,2,3, \ldots$, and suppose that

$$
\gamma_{n}^{2} \geq \alpha^{2} \gamma_{n-1} \gamma_{n+1}, \quad \text { where } \alpha \geq \max \left(2, \frac{\sqrt{2}}{2}\left(1+\sqrt{1+\gamma_{1}}\right)\right) .
$$

Then $\phi(x)$ and $\tilde{\phi}(x)=\sum_{n=0}^{\infty} \gamma_{n}\left(\begin{array}{l}x \\ n\end{array}\right)$ are entire functions of order zero and $\phi, \tilde{\phi} \in \mathcal{L}$-PI.

Proof. If $\gamma_{N}=0$ for some $N>1$, then by (4.16), $\gamma_{N}=\gamma_{n+1}=\cdots=0$, so that both $\phi$ and $\tilde{\phi}$ reduce to polynomials which have only real negative zeros by Theorem 4.3. Thus, we may assume that $\gamma_{n}>0$ for all $n \geq 1$. The assertion that $\phi \in \mathcal{L}$-PI follows from a result of Hutchinson [12]. By (4.16), Lemma 4.2, and Whittaker's theorem (cf. Theorem 4.1), we know that $\tilde{\phi}$ is an entire function of order zero. In addition, by Theorem 4.3 , for each positive integer $N$, the polynomial $\tilde{\phi}_{N}(x)=\sum_{n=0}^{N} \gamma_{n}\left(\begin{array}{l}x \\ n\end{array}\right)$ has only real negative zeros. Now, another application of Whittaker's theorem shows that $\tilde{\phi}_{n} \rightarrow \tilde{\phi}$ as $N \rightarrow \infty$, uniformly on compact subsets of $\mathbb{C}$. Therefore, it follows from Hurwitz' theorem that $\tilde{\phi}$ has only real negative zeros. 
We recall that a sequence $\left\{\gamma_{k}\right\}_{k=0}^{\infty}$ is called a Laguerre sequence if it can be interpolated by a function $\phi \in \mathcal{L}-\mathcal{P}(-\infty, 0)$, that is, $\phi(k)=\gamma_{k}$ for $k=0,1,2, \ldots$ In order to expedite our exposition, we also shall introduce the following definition.

Definition 4.6. A sequence $\left\{\gamma_{k}\right\}_{k=0}^{\infty}$ of nonnegative real numbers will be called a rapidly decreasing sequence if $\left\{\gamma_{k}\right\}_{k=0}^{\infty}$ satisfies inequality (4.16).

The sequence $\left\{e^{-a k^{2}}\right\}_{k=0}^{\infty}$ is rapidly decreasing if $a \geq \log 2$ and this sequence is a Laguerre sequence for any $a>0$. Sequences of the form $\left\{e^{-a k^{p}}\right\}_{k=0}^{\infty}$ where $a>0$ and $p$ is a positive integer, $p \geq 3$, are multiplier sequences, but these sequences cannot be interpolated by functions $\phi \in \mathcal{L}-\mathcal{P}(-\infty, 0)$. Indeed, if $\phi \in \mathcal{L}-\mathcal{P}(-\infty, 0)$, then

$$
\phi(x)=e^{-\alpha x^{2}+\beta x} \Pi(x):=e^{-\alpha x^{2}+\beta x} \prod_{n=1}^{\infty}\left(1+x / x_{n}\right) e^{-x / x_{n}}
$$

where $\alpha \geq 0, \beta \in \mathbb{R}, x_{n}>0$, and $\sum_{n=1}^{\infty} 1 / x_{n}^{2}<\infty$. Then from the standard estimates of the canonical product $\Pi(x)$ (see, for example, $[1$, p. 21]), we deduce that for any $\epsilon>0$ there is a positive integer $k_{0}$ such that

$$
\Pi(k)>e^{-k^{2+\epsilon}} \quad\left(k \geq k_{0}\right) .
$$

We infer from (4.17) and (4.18) that complex zero decreasing sequences which decay at least as fast as $\left\{e^{-a k^{3}}\right\}_{k=0}^{\infty}$ cannot be interpolated by functions $\phi$ in $\mathcal{L}-\mathcal{P}(-\infty, 0)$.

By way of applications of Corollary 4.5, we next show how rapidly decreasing sequences can be used to generate complex zero decreasing sequences.

Corollary 4.7. Let $\left\{\gamma_{k}\right\}_{k=0}^{\infty}, \gamma_{0}=1, \gamma_{k}>0$, be a rapidly decreasing sequence. Then, for each fixed $t \geq \gamma_{1}$,

$$
\tilde{\phi}_{t}(x)=\sum_{j=0}^{\infty} \frac{\gamma_{j}}{t^{j}}\left(\begin{array}{l}
x \\
j
\end{array}\right) \in \mathcal{L}-\mathcal{P} \mathrm{I} .
$$

Moreover, if $T_{t}=\left\{g_{k}(1 / t)\right\}_{k=0}^{\infty}$ where $g_{k}(t)=\sum_{j=0}^{k}\left(\begin{array}{l}k \\ j\end{array}\right) \gamma_{j} t^{j}$ is the kth Jensen polynomial associated with the sequence $\left\{\gamma_{k}\right\}_{k=0}^{\infty}$, then $T_{t}$ is a CZDS for $t \geq \gamma_{1}$; that is, for any polynomial $f(x)=\sum_{k=0}^{N} a_{k} x^{k} \in \mathbb{R}[x]$, we have $Z_{c}\left(T_{t}[f(x)]\right) \leq Z_{c}(f)$ for $t \geq \gamma_{1}$ where $T_{t}[f(x)]=\sum_{0}^{N} a_{k} g_{k}(1 / t) x^{k}$.

Proof. If $\left\{\gamma_{k}\right\}_{k=0}^{\infty}$ is a rapidly decreasing sequence, then for any $t \geq \gamma_{1}$, the sequence $\left\{\gamma_{j} / t^{j}\right\}_{j=0}^{\infty}$ is also a rapidly decreasing sequence. Thus, by Corollary $4.5, \tilde{\phi}_{t}(x) \in \mathcal{L}$ - $\mathcal{P}$, so the sequence $\left\{\tilde{\phi}_{t}(k)\right\}_{k=0}^{\infty}$ is a Laguerre sequence whenever $t \geq \gamma_{1}$. Therefore, by Laguerre's theorem (see Theorem 1.4), $Z_{c}\left(\sum_{0}^{N} a_{k} \tilde{\phi}_{t}(k) x^{k}\right) \leq Z_{c}(f)$ for $t \geq \gamma_{1}$, and since $\tilde{\phi}_{t}(k)=g_{k}(1 / t)$, the corollary is established.

Corollary 4.8. Let $\left\{\gamma_{k}\right\}_{k=0}^{\infty}$ be a rapidly decreasing sequence, and let

$$
\beta_{k}=\sum_{j=0}^{k}\left(\begin{array}{l}
k \\
j
\end{array}\right) \gamma_{j}
$$

Then the sequence $\left\{\beta_{k}\right\}_{k=0}^{\infty}$ is a $C Z D S$.

Proof. Let

$$
\Delta^{k} \beta_{0}=\sum_{j=0}^{k}(-1)^{k+j}\left(\begin{array}{l}
k \\
j
\end{array}\right) \beta_{j}
$$


Then (4.19) and (4.20) are inverse relations in the sense that $\gamma_{k}=\Delta^{k} \beta_{0}[26$, p. 44]. Since $\left\{\gamma_{k}\right\}_{k=0}^{\infty}$ is a rapidly decreasing sequence, Corollary 4.5 gives that $\tilde{\phi}(x)=$ $\sum_{j=0}^{\infty} \Delta^{j} \beta_{0}\left(\begin{array}{c}x \\ j\end{array}\right) \in \mathcal{L}$-PI. Using (4.19) and $\gamma_{j}=\Delta^{j} \beta_{0}$, we obtain $\tilde{\phi}(k)=\sum_{j=0}^{k}\left(\begin{array}{l}k \\ j\end{array}\right) \Delta^{j} \beta_{0}$ $=\sum_{j=0}^{k}\left(\begin{array}{l}k \\ j\end{array}\right) \gamma_{j}=\beta_{k}$. This shows that the sequence $\left\{\beta_{k}\right\}_{k=0}^{\infty}$ is a Laguerre sequence and, consequently, also a CZDS.

Remark 4.9. We remark that if $\left\{\gamma_{0}, \gamma_{1}, \ldots, \gamma_{n}, 0,0, \ldots\right\}$ is a CZDS with $\gamma_{k}>0$ for $0 \leq k \leq n$, then the sequence $\left\{g_{k}(t)\right\}_{k=0}^{\infty}$ where $g_{k}(t)=\sum_{j=0}^{k}\left(\begin{array}{c}k \\ j\end{array}\right) \gamma_{j} t^{j}$ may not be a CZDS for some $t>0$. To verify this claim, consider the sequence $T=$ $\left\{1,1, \frac{1}{2}, 0,0, \ldots\right\}$. By Proposition 3.5, $T$ is a CZDS. A calculation shows that $g_{k}(t)=$ $1+k t+\frac{k(k-1)}{4} t^{2}$. Let $h_{t}(x)=1+x t+\frac{x(x-1)}{4} t^{2}$, so that $h_{t}(k)=g_{k}(t)$. But $h_{t}(x)$ has real zeros (both of which are positive) if and only if $t \geq 8$. Hence, by Theorem 2.13, $\left\{g_{k}(t)\right\}$ is not a CZDS for any $t>0$.

\section{A class of CZDS and a curve theorem}

In contrast to the previous examples, we will next exhibit a CZDS $\left\{\gamma_{k}\right\}_{k=0}^{\infty}$ for which the sequence $\left\{g_{k}(t)\right\}_{k=0}^{\infty}$ is a CZDS for all $t>0$, where $g_{k}(t)=\sum_{j=0}^{k}\left(\begin{array}{c}k \\ j\end{array}\right) \gamma_{j} t^{j}$. To this end, we need some preparatory results which are of independent interest.

Lemma 5.1. If $p(x)=\sum_{j=0}^{n} b_{j} x^{j} \in \mathcal{L}-\mathcal{P}(-\infty,-1]$, then we obtain $\phi(x)=\sum_{j=0}^{n} b_{j}\left(\begin{array}{l}x \\ j\end{array}\right)$ $\in \mathcal{L}-\mathcal{P}(-\infty,-1]$

Proof. Let $T_{B}, T_{\Gamma}: \mathbb{R}[x] \rightarrow \mathbb{R}[x]$ denote the linear operators defined by

$$
T_{B}\left[\sum_{k=0}^{n} c_{k} x^{k}\right]:=\sum_{k=0}^{n} c_{k} x(x-1) \cdots(x-k+1)
$$

and

$$
T_{\Gamma}\left[\sum_{k=0}^{n} c_{k} x^{k}\right]:=\sum_{k=0}^{n} \frac{c_{k}}{k !} x^{k},
$$

respectively. Set $p_{1}(x):=p(x-1)$, so that $p_{1}(x)=\sum_{k=0}^{n} c_{k} x^{k} \in \mathcal{L}-\mathcal{P}(-\infty, 0]$. Since $\left\{\frac{1}{k !}\right\}_{k=0}^{\infty}$ is a CZDS, it follows that

$$
T_{\Gamma}\left[p_{1}(x)\right]=T_{\Gamma}[p(x-1)]=\sum_{k=0}^{n} \frac{c_{k}}{k !} x^{k} \in \mathcal{L}-\mathcal{P}(-\infty, 0] .
$$

Next, a straightforward induction argument shows that

$$
T_{B}\left[\sum_{j=0}^{k}\left(\begin{array}{l}
k \\
j
\end{array}\right) \frac{x^{j}}{j !}\right]=\frac{1}{k !} \prod_{j=1}^{k}(x+j) .
$$

Then, by the linearity of $T_{B}, T_{\Gamma}$, and the above formula, we have

$$
\begin{aligned}
p_{2}(x) & :=T_{B}\left[T_{\Gamma}\left[p_{1}(x+1)\right]\right]=T_{B}\left[T_{\Gamma}\left[\sum_{k=0}^{n} c_{k}(x+1)^{k}\right]\right] \\
& =T_{B}\left[\sum_{k=0}^{n} c_{k} T_{\Gamma}\left[(x+1)^{k}\right]\right]=T_{B}\left[\sum_{k=0}^{n} c_{k}\left(\sum_{j=0}^{k}\left(\begin{array}{l}
k \\
j
\end{array}\right) \frac{x^{j}}{j !}\right)\right] \\
& =\sum_{k=0}^{n} c_{k} T_{B}\left[\sum_{j=0}^{k}\left(\begin{array}{l}
k \\
j
\end{array}\right) \frac{x^{j}}{j !}\right]=\sum_{k=0}^{n} \frac{c_{k}}{k !} \prod_{j=1}^{k}(x+j) .
\end{aligned}
$$


Thus,

$$
p_{2}(x-1)=\sum_{k=0}^{n} \frac{c_{k}}{k !} \prod_{j=0}^{k-1}(x+j) .
$$

Since $\sum_{k=0}^{n} c_{k} x^{k} / k ! \in \mathcal{L}-\mathcal{P}(-\infty, 0]$ by $(5.1)$, it follows from a result of [2, p. 18] or [25, II, Part V, Ch. 3, Problem 185] that $p_{2}(x-1) \in \mathcal{L}-\mathcal{P}(-\infty, 0]$. Consequently,

$$
\begin{aligned}
p_{2}(x) & =T_{B}\left[T_{\Gamma}\left[p_{1}(x+1)\right]\right]=T_{B}\left[T_{\Gamma}[p(x)]\right] \\
& =\phi(x)=\sum_{j=0}^{n} b_{j}\left(\begin{array}{l}
x \\
j
\end{array}\right) \in \mathcal{L}-\mathcal{P}(-\infty,-1] .
\end{aligned}
$$

Lemma 5.2. For each fixed $t>0$,

$$
\tilde{I}_{t}(x):=\sum_{j=0}^{\infty} \frac{t^{j}}{j !}\left(\begin{array}{l}
x \\
j
\end{array}\right) \in \mathcal{L}-\mathcal{P}(-\infty,-1] .
$$

Proof. Fix $t>0$. Then $\varlimsup_{k \rightarrow \infty}\left|t^{k} / k !\right|^{1 / k}=0$, so by Whittaker's theorem (see Theorem 4.1), $\tilde{I}_{t}(x)$ is an entire function. Next, consider the polynomial $p_{n, t}(x)=$ $\sum_{k=0}^{n}\left(\begin{array}{l}n \\ k\end{array}\right) x^{k} t^{k} / n^{k}=(1+x t / n)^{n}$, whose zeros are $x=-n / t<-1$ provided $n>t$. Therefore, by Lemma 5.1, for $n>t$,

$$
\tilde{p}_{n, t}(x):=\sum_{k=0}^{n}\left(\begin{array}{l}
n \\
k
\end{array}\right) \frac{t^{k}}{n^{k}}\left(\begin{array}{l}
x \\
k
\end{array}\right) \in \mathcal{L}-\mathcal{P}(-\infty,-1] .
$$

Since $\tilde{I}_{t}(x)$ is an entire function, an argument similar to the one used in Corollary 4.5 shows that $\tilde{p}_{n, t}(x)$ converges as $n \rightarrow \infty$ uniformly on compact subsets of $\mathbb{C}$, to $\tilde{I}_{t}(x)$. Therefore, $\tilde{I}_{t}(x) \in \mathcal{L}-\mathcal{P}(-\infty,-1]$.

Lemma 5.3. Let $\gamma_{k}=1 / k !, k=0,1,2, \ldots$. Then $\left\{\gamma_{k}\right\}_{k=0}^{\infty}$ is a CZDS, and for each fixed $t>0,\left\{g_{k}(t)\right\}_{k=0}^{\infty}$ is a CZDS.

Proof. The assertion that $\{1 / k !\}_{k=0}^{\infty}$ is a CZDS is well-known [20, Satz 5.8]. Let $f(x)=\sum_{k=0}^{N} a_{k} x^{k} \in \mathbb{R}[x]$ be an arbitrary polynomial. Now, by Lemma 5.2 , the entire function $\tilde{I}_{t}(x) \in \mathcal{L}-\mathcal{P}(-\infty,-1]$ and $\tilde{I}_{t}(k)=g_{k}(t)$ for each fixed $t>0$. Hence, by Laguerre's theorem, $Z_{c}\left(\sum_{k=0}^{N} a_{k} g_{k}(t) x^{k}\right) \leq Z_{c}(f)$, so that $\left\{g_{k}(t)\right\}_{k=0}^{\infty}$ is a CZDS.

Theorem 5.4. Let $\left\{\gamma_{k}\right\}_{k=0}^{\infty}$ be a CZDS, and suppose that for each fixed $t>0$, $\left\{g_{k}(t)\right\}_{k=0}^{\infty}$ is a CZDS where $g_{k}(t)=\sum_{j=0}^{k}\left(\begin{array}{l}k \\ j\end{array}\right) \gamma_{j} t^{j}$. Suppose that the polynomial $f(x)=\sum_{k=0}^{n} a_{k} x^{k} / k ! \in \mathbb{R}[x], a_{n} \neq 0$, has exactly $r$ real zeros counting multiplicities. Consider the function $F(x, y):=\sum_{k=0}^{n} \gamma_{k} x^{k} f^{(k)}(y) / k !$. Then the curve $F(x, y)=0$ intersects each line $y=x / s, s>0$, in at least $r$ (real) points.

Proof. A calculation shows that

$$
\sum_{k=0}^{n} \frac{a_{k}}{k !} g_{k}(t) x^{k}=\sum_{k=0}^{n} \frac{\gamma_{k}}{k !} x^{k} t^{k} f^{(k)}(x) .
$$

Setting $x=y$ and then $t=x / y$ in this equation, we obtain

$$
\sum_{k=0}^{n} \frac{a_{k}}{k !} g_{k}(x / y) y^{k}=\sum_{k=0}^{n} \frac{\gamma_{k}}{k !} x^{k} f^{(k)}(y)=F(x, y) .
$$


Fix $s>0$. Then the points of intersection of $F(x, y)=0$ with the line $y=x / s$ are the real zeros of the polynomial $H(y)=\sum_{k=0}^{n} \gamma_{k}(s y)^{k} f^{(k)}(y) / k$ !. Since $\left\{g_{k}(s)\right\}_{k=0}^{\infty}$ is a CZDS for all $s>0$, we have $Z_{c}\left(\sum_{k=0}^{n} a_{k} g_{k}(s) x^{k} / k !\right) \leq Z_{c}(f)=n-r$, and consequently the polynomial $H(y)$ has at least $r$ real zeros.

Acknowledgement. The authors wish to thank the referees for their careful reading of the manuscript.

\section{References}

1. R. P. Boas, Jr., Entire Functions, Academic Press, New York, 1954.

2. F. Brenti, Unimodal, Log-Concave and Pólya Frequency Sequences in Combinatorics, Memoirs of the Amer. Math Soc., Vol. 81, No. 413, Providence, RI, 1989.

3. T. Craven and G. Csordas, Multiplier sequences for fields, Illinois J. Math. 21 (1977), 801-817.

4. - Zero-diminishing linear transformations, Proc. Amer. Math. Soc. 80 (1980), 544-546.

5. - An inequality for the distribution of zeros of polynomials and entire functions, Pacific J. Math. 95 (1981), 263-280.

6. _- On the number of real roots of polynomials, Pacific J. Math. 21 (1982), 15-28.

7. — Location of zeros, Part I: Real polynomials and entire functions, Illinois J. Math. 27 (1983), 244-278.

8. _ Location of zeros, Part II: Ordered fields, Illinois J. Math. 27 (1983), 279-299.

9. _ The Gauss-Lucas theorem and Jensen polynomials, Trans. Amer. Math. Soc. 278 (1983), $415-429$.

10. G. H. Hardy, On the zeros of a class of integral functions, Messenger of Math. 34 (1904), 97-101.

11. _ Collected Papers of G. H. Hardy, Vol. IV, Oxford Clarendon Press, 1969.

12. J. I. Hutchinson, On a remarkable class of entire functions, Trans. Amer. Math. Soc. 25 (1923), 325-332.

13. L. Iliev Laguerre Entire Functions, Pub. House of the Bulgarian Acad. Sci., Sofia, 1987.

14. A. Iserles, S. P. Nørsett, and E. B. Saff, On transformations and zeros of polynomials, Rocky Mountain J. Math. 21 (1991), 331-357.

15. S. Karlin, Total Positivity, Vol. 1, Stanford Univ. Press, Stanford, Calif., 1968.

16. M. D. Kostova, Über die $\lambda$-Folgen, C. R. Acad. Bulgare Sci. 36 (1983), 23-25.

17. D. C. Kurtz, A sufficient condition for all the roots of a polynomial to be real, Amer. Math. Monthly 99 (1992), 259-263.

18. M. Marden, Geometry of Polynomials, Math. Surveys No. 3, Amer. Math. Soc., Providence, RI, 1966.

19. I. P. Natanson, Constructive Function Theory, Vol. II, Approximation in Mean, (transl. by J. R. Schulenberger), Fredrick Ungar Pub. Co., New York, 1965.

20. N. Obreschkoff, Verteilung und Berechnung der Nullstellen reeller Polynome, VEB Deutscher Verlag der Wissenschaften, Berlin, 1963.

21. Petrovitch, Une classe remarquable de séries entières, Atti del IV Congresso Internationale dei Matematici, Rome, Ser. 1, 2 (1908), 36-43.

22. G. Pólya, Über einen Satz von Laguerre, Jber. Deutsch. Math-Verein. 38 (1929), 161-168.

23. —_ Collected Papers, Vol. II, Location of Zeros, (R. P. Boas, ed.), MIT Press, Cambridge, MA, 1974.

24. G. Pólya and J. Schur, Über zwei Arten von Faktorenfolgen in der Theorie der algebraischen Gleichungen, J. Reine Angew. Math. 144 (1914), 89-113.

25. G. Pólya and G. Szegö, Problems and Theorems in Analysis, Vols. I and II, Springer-Verlag, New York, 1976.

26. J. Riordan, Combinatorial Identities, John Wiley, New York, 1968.

27. L. Weisner, Roots of certain classes of polynomials, Bull. Amer Math. Soc. 48 (1942), 283-286.

28. J. M. Whittaker, Interpolatory Function Theory, Cambridge Tracts in Mathematics and Mathematical Physics, No. 33, Cambridge Univ. Press, Cambridge, 1935.

29. D. V. Widder, The Laplace Transform, Princeton Univ. Press, Princeton, 1941.

Department of Mathematics, University of Hawail, Honolulu, Hi 96822

E-mail: tom@math.hawaii.edu, george@math.hawaii.edu 